\title{
Geometrical scaling for energies available at the BNL Relativistic Heavy Ion Collider to those at the CERN Large Hadron Collider
}

\author{
M. Petrovici, ${ }^{1,2, *}$ A. Lindner ${ }^{1,2}$ A. Pop, ${ }^{1}$ M. Târzilă ${ }^{1,2}$ and I. Berceanu ${ }^{1}$ \\ ${ }^{1}$ National Institute for Physics and Nuclear Engineering - IFIN-HH, Hadron Physics Department, Bucharest, Romania \\ ${ }^{2}$ Faculty of Physics, University of Bucharest, Romania
}

(Received 11 May 2018; published 7 August 2018)

\begin{abstract}
Based on recent experimental results at the BNL Relativistic Heavy Ion Collider (RHIC) and the CERN Large Hadron Collider (LHC), the $\left\langle p_{T}\right\rangle$ dependence of identified light flavor charged hadrons on $\sqrt{\left(\frac{d N}{d y}\right) / S_{\perp}}$, the relevant scale in the gluon saturation picture, is studied from $\sqrt{s_{N N}}=7.7 \mathrm{GeV}$ up to $5.02 \mathrm{TeV}$. This study is extended to the slopes of the $\left\langle p_{T}\right\rangle$ dependence on the particle mass and the $\left\langle\beta_{T}\right\rangle$ parameter from Boltzmann-Gibbs blast wave (BGBW) fits of the $p_{T}$ spectra. A systematic decrease of the slope of the $\left\langle p_{T}\right\rangle$ dependence on $\sqrt{\left(\frac{d N}{d y}\right) / S_{\perp}}$ from Beam Energy Scan (BES) to LHC energies is evidenced. While, for the RHIC energies, within the experimental errors, $\left\langle p_{T}\right\rangle / \sqrt{\left(\frac{d N}{d y}\right) / S_{\perp}}$ does not depend on centrality, at the LHC energies a deviation from a linear behavior is observed towards the most central collisions. The influence of the corona contribution on the observed trends is discussed. The slopes of the $\left\langle p_{T}\right\rangle$ particle mass dependence and the $\left\langle\beta_{T}\right\rangle$ parameter from BGBW fits scale well with $\sqrt{\left(\frac{d N}{d y}\right) / S_{\perp}}$. Similar systematic trends for $p p$ at $\sqrt{s}=7 \mathrm{TeV}$ are in a good agreement with the ones corresponding to $\mathrm{Pb}-\mathrm{Pb}$ collisions at $\sqrt{s_{N N}}=2.76$ and $5.02 \mathrm{TeV}$, pointing to a system-size-independent behavior.
\end{abstract}

\section{DOI: 10.1103/PhysRevC.98.024904}

\section{INTRODUCTION}

The parton density evolution as a function of $x$ and $Q^{2}$, addressed more than 35 years ago [1], and its experimental confirmation at the Hadron-Electron Ring Accelerator (HERA) at DESY [2] have triggered a real interest in the community studying ultrarelativistic heavy ion collisions. The rise of the structure function at low $\mathrm{x}$, still visible at small values of $Q^{2}$ $[3,4]$ where the perturbative QCD does not work anymore, requires new approaches for a complete understanding of the $\log \frac{1}{x}-\log Q^{2}$ QCD landscape. Low $x$ values and moderate $Q^{2}$ are characteristic features for the early stage of hadron collisions starting from energies available at the BNL Relativistic Heavy Ion Collider (RHIC) up to those available at the CERN Large Hadron Collider (LHC). For average transverse momentum $\left(\left\langle p_{T}\right\rangle\right)$ values of the order of $1-2 \mathrm{GeV} / c$, specific for this range of energies, the $x$ values at mid-rapidity are of the order of $\sim 10^{-2}$ and $\sim 10^{-4}$ respectively. Such initial conditions are used by different theoretical approaches for describing especially the most recent results from LHC energies. Color glass condensate (CGC) is one of such approaches, based on a strong classical color fields description of the small- $x$ degrees of freedom [5-7]. The local parton-hadron duality picture (LPHD) [8] and dimensionality argument $[9,10]$ predict a decrease of the ratio between the average transverse momentum and

"mpetro@nipne.ro

Published by the American Physical Society under the terms of the Creative Commons Attribution 4.0 International license. Further distribution of this work must maintain attribution to the author $(s)$ and the published article's title, journal citation, and DOI. Funded by $S C O A P^{3}$. the square root of the hadron multiplicity per unit of rapidity and unit of the colliding hadrons' transverse overlapping area $\left[\left\langle p_{T}\right\rangle / \sqrt{(d N / d y) / S_{\perp}}\right]$ towards central collisions and higher energies. With the latest results from the Beam Energy Scan (BES) at RHIC and the highest energies at LHC, it is worth revisiting such a dependence. Recently evidenced similarities between $p p, p-\mathrm{Pb}$ and $\mathrm{Pb}-\mathrm{Pb}$ collisions at $\mathrm{LHC}$ energies in terms of long-range near-side two-particle correlations, transverse flow, and strangeness enhancement as a function of charged particle multiplicity [11-17] support the idea that even in small colliding systems, due to increased parton density at such energies, the probability of multiple parton interaction increases, the rescattering processes become important, and a thermalized stage could be reached although the interaction time is extremely short. Such a high density deconfined small system could follow a hydrodynamic type expansion. To what extent the hydrodynamics is applicable in small systems is still under debate [18]. The most successful phenomenological models, URQMD, HIJING, NEXSPHERIO, AMPT, PHSD, EPOS, describing the latest results obtained at LHC in $p p, p-\mathrm{Pb}$ and $\mathrm{Pb}-\mathrm{Pb}$ collisions are based on combinations of different approaches for different stages of the collision [19-24], while the classical phenomenological models used in particle physics like PYTHIA [25], HERWIG [26], and PHOJET [27] had to implement processes like multiparton interaction, rescattering, color reconnection [28], or a shoving mechanism [29] in order to improve the agreement with the LHC results, especially in the soft sector in $p p$ collisions. In this paper we also present a comparison between $p p$ and $\mathrm{Pb}-\mathrm{Pb}$ at LHC energies in terms of the dependence of different observables on the $\sqrt{(d N / d y) / S_{\perp}}$ variable. In Sec. II the estimates of the overlapping area of the colliding hadrons are presented. Details on the hadron density per unit of rapidity are given in Sec. III. The $\left\langle p_{T}\right\rangle$ dependence 
on $\sqrt{(d N / d y) / S_{\perp}}$ is presented in Sec. IV for BES and $\sqrt{s_{N N}}=$ 62.4, 130, $200 \mathrm{GeV}$ Au-Au collisions measured by the STAR Collaboration at RHIC and for $\mathrm{Pb}-\mathrm{Pb}$ collisions at $\sqrt{s_{N N}}=$ 2.76 and $5.02 \mathrm{TeV}$ measured by the ALICE Collaboration at LHC. Section V is dedicated to the $\sqrt{(d N / d y) / S_{\perp}}$ dependence of the slope of the linear $\left\langle p_{T}\right\rangle$ versus particle mass behavior for identified light flavor charged hadrons. The Boltzmann-Gibbs blast wave (BGBW) fit parameters of $p_{T}$ spectra are presented versus the same geometrical variable of the colliding systems in Sec. VI. Similarities, in terms of $\sqrt{(d N / d y) / S_{\perp}}$ dependence of different observables, in $p p$ at $\sqrt{s}=7 \mathrm{TeV}$ and $\mathrm{Pb}-\mathrm{Pb}$ at $\sqrt{s_{N N}}=2.76$ and $5.02 \mathrm{TeV}$ are discussed in Sec. VII. Section VIII is dedicated to conclusions.

\section{OVERLAPPING AREA $S_{\perp}$ ESTIMATES}

The overlapping area of the two colliding nuclei for a given incident energy and centrality was estimated based on the Glauber Monte Carlo (GMC) approach [30-33]. For the nuclear density profile of the colliding nuclei, a Woods-Saxon distribution was considered:

$$
\rho(r)=\frac{1}{1+\exp \left(\frac{r-r_{0}}{a}\right)},
$$

with $a=0.535 \mathrm{fm}, r_{0}=6.5 \mathrm{fm}$ for the Au nucleus [34] and $a=0.546 \mathrm{fm}, r_{0}=6.62 \mathrm{fm}$ for the Pb nucleus [35]. Within the black disk approach, the nucleons are considered to collide

TABLE I. For the colliding systems Au-Au at $\sqrt{s_{N N}}=7.7,11.5,19.6,27$, and $39 \mathrm{GeV}$ from BES [38], Au-Au at $\sqrt{s_{N N}}=62.4$, 130, and $200 \mathrm{GeV}$ [34] studied at RHIC by the STAR Collaboration, and Pb-Pb at $\sqrt{s_{N N}}=2.76$ and $5.02 \mathrm{TeV}$ investigated by the ALICE Collaboration at LHC [35,37], we show the colliding system, the collision energy, the centrality, the average number of participant nucleons in the collision $\left(\left\langle N_{\text {part }}\right\rangle\right)$, the overlapping areas corresponding to the wounded nucleons $\left(S_{\perp}^{\text {geom }}, S_{\perp}^{\text {var }}\right)$ estimated by the two recipes explained in the text, the percentage of the wounded nucleons undergoing more than a single collision $\left(f_{\text {core }}\right)$, the corresponding areas of the wounded nucleons undergoing more than a single collision $\left[\left(S_{\perp}^{\text {geom }}\right)^{\text {core }},\left(S_{\perp}^{\text {var }}\right)^{\text {core }}\right]$, and the hadron density $(d N / d y)$.

\begin{tabular}{|c|c|c|c|c|c|c|c|c|c|}
\hline System & $\begin{array}{l}\sqrt{s_{N N}} \\
(\mathrm{GeV})\end{array}$ & $\begin{array}{l}\text { Cen. } \\
(\%)\end{array}$ & $\left\langle N_{\text {part }}\right\rangle$ & $\begin{array}{l}S_{\perp}^{\text {geom }} \\
\left(\mathrm{fm}^{2}\right)\end{array}$ & $\begin{array}{c}S_{\perp}^{\text {var }} \\
\left(\mathrm{fm}^{2}\right)\end{array}$ & $f_{\text {core }}$ & $\begin{array}{c}\left(S_{\perp}^{\text {geom }}\right)^{\text {core }} \\
\left(\mathrm{fm}^{2}\right)\end{array}$ & $\begin{array}{c}\left(S_{\perp}^{\mathrm{var}}\right)^{\text {core }} \\
\left(\mathrm{fm}^{2}\right)\end{array}$ & $d N / d y$ \\
\hline \multirow[t]{9}{*}{$\mathrm{Au}-\mathrm{Au}$} & \multirow[t]{9}{*}{7.7} & $0-5$ & $337 \pm 2$ & $146.1 \pm 0.7$ & $147.1 \pm 0.7$ & $0.88 \pm 0.00$ & $126.5 \pm 0.6$ & $124.6 \pm 0.6$ & $476.7 \pm 22.5$ \\
\hline & & $5-10$ & $0 \pm 6$ & $126.9 \pm 0.7$ & $129.7 \pm 0.6$ & $0.84 \pm 0.00$ & $107.6 \pm 0.7$ & $105.0 \pm 0.5$ & $392.5 \pm 18.5$ \\
\hline & & $10-20$ & $26 \pm 8$ & $103.6 \pm 0.7$ & $108.9 \pm 0.5$ & $0.80 \pm 0.00$ & $85.5 \pm 0.7$ & $84.3 \pm 0.4$ & $295.4 \pm 14.0$ \\
\hline & & $20-30$ & $160 \pm 10$ & $79.7 \pm 0.8$ & $87.1 \pm 0.4$ & $0.75 \pm 0.00$ & $63.3 \pm 0.7$ & $63.8 \pm 0.3$ & $03.6 \pm 9.8$ \\
\hline & & $30-40$ & $110 \pm 11$ & $61.2 \pm 0.8$ & $70.4 \pm 0.3$ & $0.70 \pm 0.00$ & $46.6 \pm 0.7$ & $48.9 \pm 0.2$ & $135.1 \pm 6.4$ \\
\hline & & $40-50$ & $72 \pm 10$ & $45.9 \pm 0.8$ & $56.9 \pm 0.3$ & $0.63 \pm 0.00$ & $33.1 \pm 0.8$ & $37.5 \pm 0.2$ & $84.8 \pm 4.1$ \\
\hline & & $50-60$ & $45 \pm 9$ & $32.8 \pm 0.8$ & $45.7 \pm 0.2$ & $0.56 \pm 0.00$ & $21.9 \pm 0.8$ & $28.9 \pm 0.1$ & $51.5 \pm 2.5$ \\
\hline & & $60-70$ & $26 \pm 6$ & $21.8 \pm 0.8$ & $36.3 \pm 0.2$ & $0.47 \pm 0.00$ & $12.8 \pm 0.7$ & $22.6 \pm 0.1$ & $27.6 \pm 1.4$ \\
\hline & & $70-80$ & $14 \pm 4$ & $12.1 \pm 0.7$ & $26.8 \pm 0.2$ & $0.37 \pm 0.00$ & $5.4 \pm 0.6$ & $15.6 \pm 0.1$ & $13.8 \pm 0.8$ \\
\hline \multirow[t]{9}{*}{$\mathrm{Au}-\mathrm{Au}$} & \multirow[t]{9}{*}{11.5} & $0-5$ & $338 \pm 2$ & $146.1 \pm 0.7$ & $147.1 \pm 0.7$ & $0.88 \pm 0.00$ & $126.5 \pm 0.6$ & $124.5 \pm 0.6$ & $565.1 \pm 29.5$ \\
\hline & & $5-10$ & $1 \pm 6$ & $126.6 \pm 0.7$ & $129.5 \pm 0.6$ & $0.84 \pm$ & $107.2 \pm 0.7$ & $104.6 \pm 0.5$ & $449.0 \pm$ \\
\hline & & $10-20$ & $226 \pm 8$ & $103.5 \pm 0.7$ & $108.9 \pm 0.5$ & $0.80 \pm 0.00$ & $85.3 \pm 0.7$ & $84.1 \pm 0.4$ & $335.5 \pm 17.5$ \\
\hline & & $20-30$ & $160 \pm 9$ & $79.9 \pm 0.8$ & $87.3 \pm 0.4$ & $0.75 \pm 0.00$ & $63.4 \pm 0.7$ & $63.9 \pm 0.3$ & $225.5 \pm 11.8$ \\
\hline & & $30-40$ & $110 \pm 10$ & $61.3 \pm 0.8$ & $70.5 \pm 0.3$ & $0.70 \pm 0.00$ & $46.6 \pm 0.7$ & $48.9 \pm 0.2$ & $152.0 \pm 8.1$ \\
\hline & & $40-50$ & $73 \pm 10$ & $45.8 \pm 0.8$ & $56.9 \pm 0.3$ & $0.63 \pm 0.00$ & $33.0 \pm 0.8$ & $37.5 \pm 0.2$ & $94.5 \pm 5.1$ \\
\hline & & $50-60$ & $44 \pm 9$ & $32.9 \pm 0.8$ & $45.9 \pm 0.2$ & $0.56 \pm 0.00$ & $21.9 \pm 0.8$ & $28.9 \pm 0.1$ & $55.8 \pm 3.1$ \\
\hline & & $60-70$ & $26 \pm 7$ & $21.8 \pm 0.8$ & $36.4 \pm 0.2$ & $0.47 \pm 0.01$ & $12.8 \pm 0.7$ & $22.6 \pm 0.1$ & $31.3 \pm 1.8$ \\
\hline & & $70-80$ & $14 \pm 6$ & $12.1 \pm 0.7$ & $26.8 \pm 0.2$ & $0.37 \pm 0.01$ & $5.4 \pm 0.6$ & $15.5 \pm 0.1$ & $16.0 \pm 0.9$ \\
\hline \multirow[t]{9}{*}{$\mathrm{Au}-\mathrm{Au}$} & \multirow[t]{9}{*}{19.6} & $0-5$ & $338 \pm 2$ & $146.6 \pm 0.7$ & $147.5 \pm 0.7$ & $0.89 \pm 0.00$ & $126.9 \pm 0.6$ & $125.0 \pm 0.6$ & $683.4 \pm 40.0$ \\
\hline & & $5-10$ & $289 \pm 6$ & $127.2 \pm 0.7$ & $129.9 \pm 0.6$ & $0.85 \pm 0.00$ & $107.6 \pm 0.7$ & $105.1 \pm 0.5$ & $556.5 \pm 32.3$ \\
\hline & & $10-20$ & $225 \pm 9$ & $104.0 \pm 0.7$ & $109.4 \pm 0.5$ & $0.81 \pm 0.00$ & $85.6 \pm 0.7$ & $84.5 \pm 0.4$ & $421.7 \pm 24.7$ \\
\hline & & $20-30$ & $158 \pm 10$ & $80.2 \pm 0.8$ & $87.7 \pm 0.4$ & $0.76 \pm 0.00$ & $63.6 \pm 0.7$ & $64.2 \pm 0.3$ & $284.3 \pm 16.6$ \\
\hline & & $30-40$ & $108 \pm 11$ & $61.4 \pm 0.8$ & $70.7 \pm 0.3$ & $0.70 \pm 0.00$ & $46.6 \pm 0.7$ & $49.0 \pm 0.2$ & $187.9 \pm 11.1$ \\
\hline & & $40-50$ & $71 \pm 10$ & $46.0 \pm 0.8$ & $57.1 \pm 0.3$ & $0.64 \pm 0.00$ & $33.0 \pm 0.8$ & $37.5 \pm 0.2$ & $117.3 \pm 7.0$ \\
\hline & & $50-60$ & $44 \pm 9$ & $32.9 \pm 0.8$ & $45.9 \pm 0.2$ & $0.56 \pm 0.00$ & $21.8 \pm 0.8$ & $28.8 \pm 0.1$ & $70.3 \pm 4.2$ \\
\hline & & $60-70$ & $25 \pm 7$ & $21.9 \pm 0.8$ & $36.6 \pm 0.2$ & $0.47 \pm 0.01$ & $12.9 \pm 0.7$ & $22.7 \pm 0.1$ & $38.1 \pm 2.3$ \\
\hline & & $70-80$ & $14 \pm 5$ & $12.1 \pm 0.7$ & $26.7 \pm 0.2$ & $0.37 \pm 0.01$ & $5.4 \pm 0.6$ & $15.5 \pm 0.1$ & $19.8 \pm 1.3$ \\
\hline \multirow[t]{7}{*}{$\mathrm{Au}-\mathrm{Au}$} & \multirow[t]{7}{*}{27} & $0-5$ & $343 \pm 2$ & $147.2 \pm 0.7$ & $148.3 \pm 0.7$ & $0.89 \pm 0.00$ & $127.6 \pm 0.6$ & $125.8 \pm 0.6$ & $727.0 \pm 42.2$ \\
\hline & & & $299 \pm 6$ & $127.8 \pm 0.7$ & $130.9 \pm 0.6$ & $0.85 \pm 0.00$ & $108.1 \pm 0.7$ & $105.9 \pm 0.5$ & $6057+25$ \\
\hline & & $10-20$ & $234 \pm 9$ & $104.6 \pm 0.7$ & $110.2 \pm 0.5$ & $0.81 \pm$ & $85.9 \pm 0.7$ & $85.1 \pm 0.4$ & $457.6 \pm 26.6$ \\
\hline & & $20-30$ & $166 \pm 11$ & $80.7 \pm 0.8$ & $88.4 \pm 0.4$ & $0.76 \pm 0.00$ & $63.8 \pm 0.7$ & $64.6 \pm 0.3$ & $309.6 \pm 18.1$ \\
\hline & & $30-40$ & $114 \pm 11$ & $61.9 \pm 0.8$ & $71.3 \pm 0.3$ & $0.71 \pm 0.00$ & $46.8 \pm 0.7$ & $49.3 \pm 0.2$ & $203.6 \pm 11.9$ \\
\hline & & $40-50$ & $75 \pm 10$ & $46.1 \pm 0.8$ & $57.4 \pm 0.3$ & $0.64 \pm 0.00$ & $33.1 \pm 0.8$ & $37.6 \pm 0.2$ & $128.2 \pm 7.6$ \\
\hline & & $50-60$ & $47 \pm 9$ & $32.9 \pm 0.8$ & $46.1 \pm 0.2$ & $0.56 \pm 0.00$ & $21.7 \pm 0.8$ & $28.8 \pm 0.1$ & $76.0 \pm 4.5$ \\
\hline
\end{tabular}


TABLE I. (Continued.)

\begin{tabular}{|c|c|c|c|c|c|c|c|c|c|}
\hline System & $\begin{array}{l}\sqrt{s_{N N}} \\
(\mathrm{GeV})\end{array}$ & $\begin{array}{l}\text { Cen. } \\
(\%)\end{array}$ & $\left\langle N_{\text {part }}\right\rangle$ & $\begin{array}{l}S_{\perp}^{\text {geom }} \\
\left(\mathrm{fm}^{2}\right)\end{array}$ & $\begin{array}{c}S_{\perp}^{\mathrm{var}} \\
\left(\mathrm{fm}^{2}\right)\end{array}$ & $f_{\text {core }}$ & $\begin{array}{c}\left(S_{\perp}^{\mathrm{geom}}\right)^{\text {core }} \\
\left(\mathrm{fm}^{2}\right)\end{array}$ & $\begin{array}{c}\left(S_{\perp}^{\mathrm{var}}\right)^{\mathrm{core}} \\
\left(\mathrm{fm}^{2}\right)\end{array}$ & $d N / d y$ \\
\hline & & $60-70$ & $27 \pm 8$ & $21.9 \pm 0.8$ & $36.6 \pm 0.2$ & $0.47 \pm 0.01$ & $12.8 \pm 0.7$ & $22.5 \pm 0.1$ & $41.9 \pm 2.3$ \\
\hline \multirow[t]{8}{*}{$\mathrm{Au}-\mathrm{Au}$} & 39 & $0-5$ & $342 \pm 2$ & $147.9 \pm 0.7$ & $149.2 \pm 0.7$ & $0.89 \pm 0.00$ & $128.4 \pm 0.6$ & $126.7 \pm 0.6$ & $756.2 \pm 44.1$ \\
\hline & & $5-10$ & $294 \pm 6$ & $128.4 \pm 0.7$ & $131.7 \pm 0.6$ & $0.86 \pm 0.00$ & $108.6 \pm 0.7$ & $106.6 \pm 0.5$ & $633.0 \pm 36.8$ \\
\hline & & $10-20$ & $230 \pm 9$ & $104.9 \pm 0.7$ & $110.8 \pm 0.5$ & $0.81 \pm 0.00$ & $86.2 \pm 0.7$ & $85.6 \pm 0.4$ & $482.2 \pm 28.2$ \\
\hline & & $30-40$ & $111 \pm 11$ & $62.2 \pm 0.8$ & $71.9 \pm 0.3$ & $0.71 \pm 0.00$ & $47.1 \pm 0.7$ & $49.7 \pm 0.2$ & $215.6 \pm 12.5$ \\
\hline & & $40-50$ & $74 \pm 10$ & $46.6 \pm 0.8$ & $58.0 \pm 0.3$ & $0.65 \pm 0.00$ & $33.3 \pm 0.8$ & $37.9 \pm 0.2$ & $136.3 \pm 7.9$ \\
\hline & & $50-60$ & $46 \pm 9$ & $33.4 \pm 0.8$ & $46.6 \pm 0.2$ & $0.57 \pm 0.00$ & $22.1 \pm 0.8$ & $29.0 \pm 0.1$ & $82.9 \pm 4.8$ \\
\hline & & $60-70$ & $26 \pm 7$ & $22.1 \pm 0.8$ & $36.8 \pm 0.2$ & $0.48 \pm 0.01$ & $12.9 \pm 0.7$ & $22.5 \pm 0.1$ & $44.9 \pm 2.6$ \\
\hline & & $70-80$ & $14 \pm 5$ & $12.8 \pm 0.7$ & $27.5 \pm 0.2$ & $0.38 \pm 0.01$ & $5.8 \pm 0.6$ & $16.1 \pm 0.1$ & $23.5 \pm 1.5$ \\
\hline \multirow[t]{6}{*}{$\mathrm{Au}-\mathrm{Au}$} & 62.4 & $0-5$ & $346.5 \pm 2.8$ & $148.9 \pm 0.7$ & $150.1 \pm 0.7$ & $0.90 \pm 0.00$ & $129.1 \pm 0.6$ & $127.6 \pm 0.6$ & $952.8 \pm 37.7$ \\
\hline & & $30-40$ & $114.3 \pm 5.1$ & $62.6 \pm 0.8$ & $72.6 \pm 0.3$ & $0.72 \pm 0.00$ & $47.1 \pm 0.7$ & $49.9 \pm 0.2$ & $270.3 \pm 11.2$ \\
\hline & & $40-50$ & $76.3 \pm 5.2$ & $47.0 \pm 0.8$ & $58.7 \pm 0.3$ & $0.65 \pm 0.00$ & $33.4 \pm 0.8$ & $38.1 \pm 0.2$ & $174.4 \pm 7.6$ \\
\hline & & $50-60$ & $47.9 \pm 4.7$ & $33.7 \pm 0.8$ & $47.1 \pm 0.2$ & $0.57 \pm 0.00$ & $22.2 \pm 0.8$ & $29.1 \pm 0.1$ & $105.5 \pm 5.1$ \\
\hline & & $60-70$ & $27.8 \pm 3.7$ & $22.3 \pm 0.8$ & $37.1 \pm 0.2$ & $0.48 \pm 0.01$ & $12.9 \pm 0.7$ & $22.4 \pm 0.1$ & $58.2 \pm 2.8$ \\
\hline & & $70-80$ & $15.3 \pm 2.4$ & $12.9 \pm 0.7$ & $27.5 \pm 0.2$ & $0.38 \pm 0.01$ & $5.8 \pm 0.7$ & $15.9 \pm 0.1$ & $28.4 \pm 1.3$ \\
\hline \multirow[t]{6}{*}{$\mathrm{Au}-\mathrm{Au}$} & 130 & $0-6$ & $344.3 \pm 3.1$ & $148.4 \pm 0.7$ & $150.3 \pm 0.7$ & $0.90 \pm 0.00$ & $128.6 \pm 0.7$ & $127.4 \pm 0.5$ & $1140.8 \pm 43.9$ \\
\hline & & $6-11$ & $289.0 \pm 5.4$ & $127.3 \pm 0.7$ & $131.8 \pm 0.6$ & $0.86 \pm 0.00$ & $106.8 \pm 0.7$ & $106.0 \pm 0.5$ & $920.6 \pm 35.1$ \\
\hline & & $11-18$ & $237.8 \pm 6.8$ & $108.7 \pm 0.7$ & $115.3 \pm 0.5$ & $0.83 \pm 0.00$ & $88.9 \pm 0.7$ & $89.1 \pm 0.4$ & $751.0 \pm 34.9$ \\
\hline & & $18-26$ & $187.7 \pm 7.5$ & $89.5 \pm 0.8$ & $97.8 \pm 0.4$ & $0.79 \pm 0.00$ & $71.0 \pm 0.7$ & $72.4 \pm 0.3$ & $569.3 \pm 24.2$ \\
\hline & & $26-34$ & $141.9 \pm 8.4$ & $72.6 \pm 0.8$ & $82.3 \pm 0.4$ & $0.75 \pm 0.00$ & $55.6 \pm 0.7$ & $58.2 \pm 0.3$ & $371.3 \pm 17.6$ \\
\hline & & $34-45$ & $100.9 \pm 8.4$ & $56.0 \pm 0.8$ & $67.2 \pm 0.3$ & $0.70 \pm 0.00$ & $40.9 \pm 0.8$ & $44.9 \pm 0.2$ & $246.0 \pm 11.0$ \\
\hline \multirow{3}{*}{$\mathrm{Au}-\mathrm{Au}$} & & $50-60$ & $49.3 \pm 4.7$ & $34.9 \pm 0.8$ & $48.9 \pm 0.2$ & $0.59 \pm 0.00$ & $22.8 \pm 0.8$ & $29.7 \pm 0.1$ & $147.8 \pm 5.9$ \\
\hline & & $60-70$ & $28.8 \pm 3.7$ & $23.8 \pm 0.8$ & $39.1 \pm 0.2$ & $0.51 \pm 0.01$ & $13.9 \pm 0.8$ & $22.7 \pm 0.1$ & $85.2 \pm 3.5$ \\
\hline & & $70-80$ & $15.7 \pm 2.6$ & $13.2 \pm 0.8$ & $28.2 \pm 0.2$ & $0.40 \pm 0.00$ & $6.2 \pm 0.7$ & $15.2 \pm 0.1$ & $42.6 \pm 1.8$ \\
\hline \multirow[t]{9}{*}{$\mathrm{Pb}-\mathrm{Pb}$} & 2760 & $0-5$ & $382.5 \pm 3.1$ & $166.9 \pm 0.7$ & $170.7 \pm 0.7$ & $0.94 \pm 0.00$ & $146.0 \pm 0.7$ & $148.0 \pm 0.6$ & $2837.0 \pm 144.0$ \\
\hline & & $5-10$ & $329.4 \pm 4.9$ & $146.1 \pm 0.7$ & $154.7 \pm 0.6$ & $0.90 \pm 0.00$ & $121.9 \pm 0.7$ & $126.5 \pm 0.5$ & $2345.5 \pm 112.4$ \\
\hline & & $10-20$ & $259.9 \pm 2.9$ & $119.8 \pm 0.8$ & $132.4 \pm 0.6$ & $0.86 \pm 0.00$ & $96.3 \pm 0.7$ & $102.7 \pm 0.4$ & $1763.2 \pm 84.8$ \\
\hline & & $20-30$ & $185.4 \pm 3.9$ & $92.9 \pm 0.8$ & $107.5 \pm 0.5$ & $0.81 \pm 0.00$ & $71.5 \pm 0.8$ & $78.4 \pm 0.3$ & $1195.8 \pm 54.2$ \\
\hline & & $30-40$ & $128.1 \pm 3.3$ & $71.4 \pm 0.8$ & $87.2 \pm 0.4$ & $0.76 \pm 0.00$ & $52.4 \pm 0.8$ & $59.7 \pm 0.2$ & $784.8 \pm 35.9$ \\
\hline & & $40-50$ & $84.2 \pm 2.6$ & $53.7 \pm 0.8$ & $70.3 \pm 0.3$ & $0.70 \pm 0.00$ & $37.2 \pm 0.8$ & $44.8 \pm 0.2$ & $482.7 \pm 21.4$ \\
\hline & & $50-60$ & $52.1 \pm 2.0$ & $38.6 \pm 0.8$ & $56.1 \pm 0.3$ & $0.63 \pm 0.00$ & $24.7 \pm 0.9$ & $33.1 \pm 0.1$ & $274.8 \pm 12.5$ \\
\hline & & $60-70$ & $29.5 \pm 1.3$ & $25.7 \pm 0.8$ & $43.6 \pm 0.2$ & $0.54 \pm 0.00$ & $14.6 \pm 0.9$ & $23.8 \pm 0.1$ & $141.8 \pm 5.4$ \\
\hline & & $70-80$ & $14.9 \pm 0.6$ & $14.2 \pm 0.8$ & $30.8 \pm 0.2$ & $0.43 \pm 0.00$ & $6.4 \pm 0.7$ & $15.1 \pm 0.1$ & $67.2 \pm 3.0$ \\
\hline \multirow[t]{9}{*}{$\mathrm{Pb}-\mathrm{Pb}$} & 5020 & $0-5$ & $385 \pm 2$ & $170.2 \pm 0.7$ & $174.2 \pm 0.7$ & $0.94 \pm 0.00$ & $149.0 \pm 0.7$ & $151.5 \pm 0.6$ & $3320.6 \pm 131.4$ \\
\hline & & $5-10$ & $333 \pm 4$ & $149.2 \pm 0.7$ & $158.5 \pm 0.6$ & $0.90 \pm 0.00$ & $124.4 \pm 0.7$ & $129.9 \pm 0.5$ & $2698.7 \pm 117.2$ \\
\hline & & $10-20$ & $263 \pm 4$ & $122.4 \pm 0.8$ & $135.8 \pm 0.6$ & $0.86 \pm 0.00$ & $98.1 \pm 0.7$ & $105.6 \pm 0.4$ & $2042.5 \pm 84.7$ \\
\hline & & $20-30$ & $188 \pm 3$ & $94.9 \pm 0.8$ & $110.5 \pm 0.5$ & $0.82 \pm 0.00$ & $72.9 \pm 0.7$ & $80.8 \pm 0.3$ & $1401.4 \pm 62.9$ \\
\hline & & $30-40$ & $131 \pm 2$ & $73.4 \pm 0.8$ & $90.0 \pm 0.4$ & $0.77 \pm 0.00$ & $53.8 \pm 0.8$ & $61.8 \pm 0.3$ & $931.0 \pm 44.5$ \\
\hline & & $40-50$ & $86.3 \pm 1.7$ & $55.7 \pm 0.8$ & $73.1 \pm 0.3$ & $0.71 \pm 0.00$ & $38.6 \pm 0.8$ & $46.9 \pm 0.2$ & $588.6 \pm 27.8$ \\
\hline & & $50-60$ & $53.6 \pm 1.2$ & $40.7 \pm 0.8$ & $58.7 \pm 0.3$ & $0.63 \pm 0.00$ & $26.3 \pm 0.8$ & $34.9 \pm 0.2$ & $346.9 \pm 26.1$ \\
\hline & & $60-70$ & $30.0 \pm 0.8$ & $27.9 \pm 0.8$ & $45.9 \pm 0.2$ & $0.54 \pm 0.01$ & $16.2 \pm 0.8$ & $25.5 \pm 0.1$ & $186.1 \pm 26.0$ \\
\hline & & $70-80$ & $15.6 \pm 0.5$ & $16.6 \pm 0.7$ & $33.0 \pm 0.2$ & $0.43 \pm 0.01$ & $7.7 \pm 0.7$ & $17.0 \pm 0.1$ & $93.5 \pm 27.4$ \\
\hline
\end{tabular}




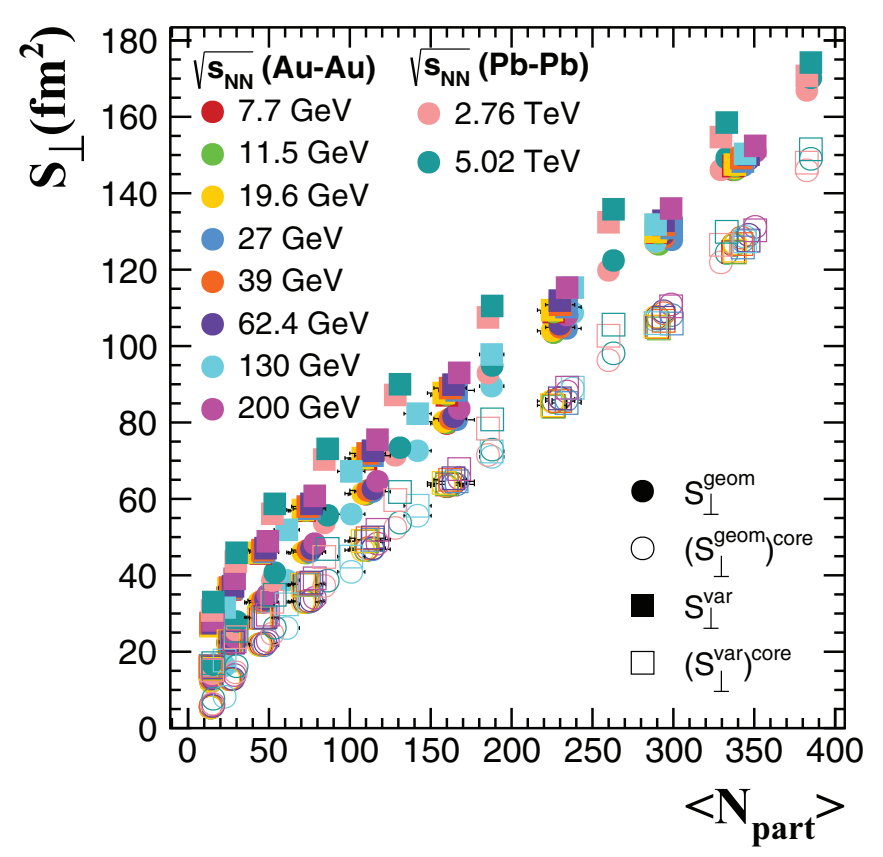

FIG. 1. Overlapping area of the colliding nuclei at different $\sqrt{s_{N N}}$ energies estimated within the GMC approach corresponding to all wounded nucleons $S_{\perp}^{\text {geom }}$ ( $S_{\perp}^{\mathrm{var}}$ ) [full dots (full squares)] and to the core contribution $\left(S_{\perp}^{\text {geom }}\right)^{\text {core }}\left(\left(S_{\perp}^{\text {var }}\right)^{\text {core }}\right)$ [open dots (open squares)] as a function of $\left\langle N_{\text {part }}\right\rangle$.

if the relative transverse distance $d \leqslant \sqrt{\frac{\sigma_{p p}}{\pi}}$, where $\sigma_{p p}$ is the nucleon-nucleon interaction cross section. The $\sigma_{p p}$ values for the corresponding $\sqrt{s_{N N}}$ energies were taken from [34-37]. The main characteristics of the collision at different centralities for Au-Au at $\sqrt{s_{N N}}=7.7,11.5,19.6,27$, and $39 \mathrm{GeV}$ obtained in the Beam Energy Scan at RHIC [38], Au-Au at $\sqrt{s_{N N}}=$ 62.4, 130, and $200 \mathrm{GeV}$ [34] and $\mathrm{Pb}-\mathrm{Pb}$ at $\sqrt{s_{N N}}=2.76$ and $5.02 \mathrm{TeV}[35,37]$ are presented in Table I (see caption for notations). The geometrical overlapping areas $\left(S_{\perp}^{\text {geom }}\right)$ have been estimated by averaging the maximum values of the $x$ and $y$ coordinates determined per event, over many events. $S_{\perp}^{\mathrm{var}}$ has been estimated as being proportional to the quantity $S=\sqrt{\left\langle\sigma_{x}^{2}\right\rangle\left\langle\sigma_{y}^{2}\right\rangle-\left\langle\sigma_{x y}\right\rangle^{2}}, \sigma_{x}^{2}$ and $\sigma_{y}^{2}$ are the variances, and $\sigma_{x y}$ is the co-variance of the participant distributions in the transverse plane, per event [39]. They were averaged $(\langle\cdots\rangle)$ over many events.

The centrality dependent values were rescaled by the factor obtained by dividing the geometrical area by $\mathrm{S}$ in the case of the complete overlap of the nuclei $(b=0 \mathrm{fm})$. The $\left\langle N_{\text {part }}\right\rangle$ dependence of the overlap area of the colliding nuclei at different energies estimated within the GMC approach corresponding to all wounded nucleons and to the core contribution are presented in Fig. 1. $\sqrt{(d N / d y) / S_{\perp}^{\text {geom }}}$ as a function of $\sqrt{s_{N N}}$ for different centralities is represented in Fig. 2. As an example, in Fig. 3 the percentage of the nucleons suffering a single collision as a function of $\left\langle N_{\text {part }}\right\rangle$ and impact parameter is represented for the lowest and highest $\sqrt{s_{N N}} \mathrm{Au}$-Au collisions, i.e., 7.7 and 200 $\mathrm{GeV}$, and for $\mathrm{Pb}-\mathrm{Pb}$ at the highest LHC energy, $\sqrt{s_{N N}}=5.02$ $\mathrm{TeV}$. As expected, the $\left\langle N_{\text {part }}\right\rangle$ dependence of the percentage

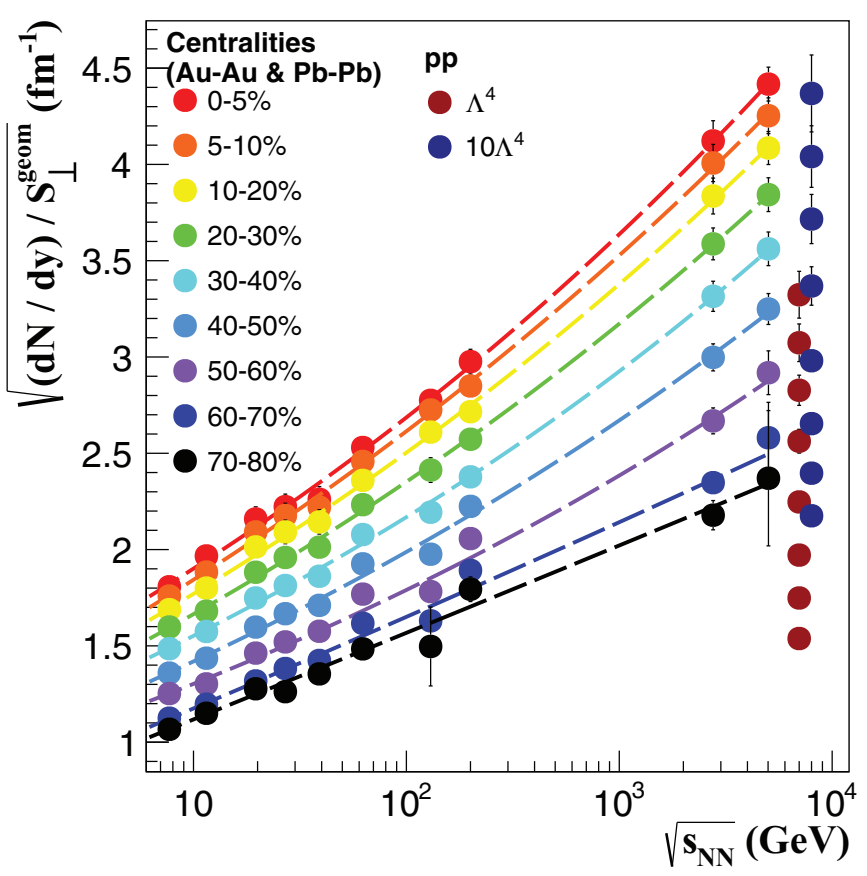

FIG. 2. $\sqrt{(d N / d y) / S_{\perp}^{\text {geom }}}$ as a function of $\sqrt{s_{N N}}$ for different centralities based on the values listed in Table I. The dashed lines represent the fit results using a power-law function. Dark red and dark blue full dots correspond to $p p$ collisions at $\sqrt{s}=7 \mathrm{TeV}$, with the values being estimated based on the IP-Glasma initial state model, using two values of the $\alpha$ parameter (see Sec. VII). For better clarity, the blue dots were artificially displaced in $\sqrt{s_{N N}}$.

of nucleons undergoing a single collision is less dependent on $\sqrt{s_{N N}}$ than on the impact parameter.

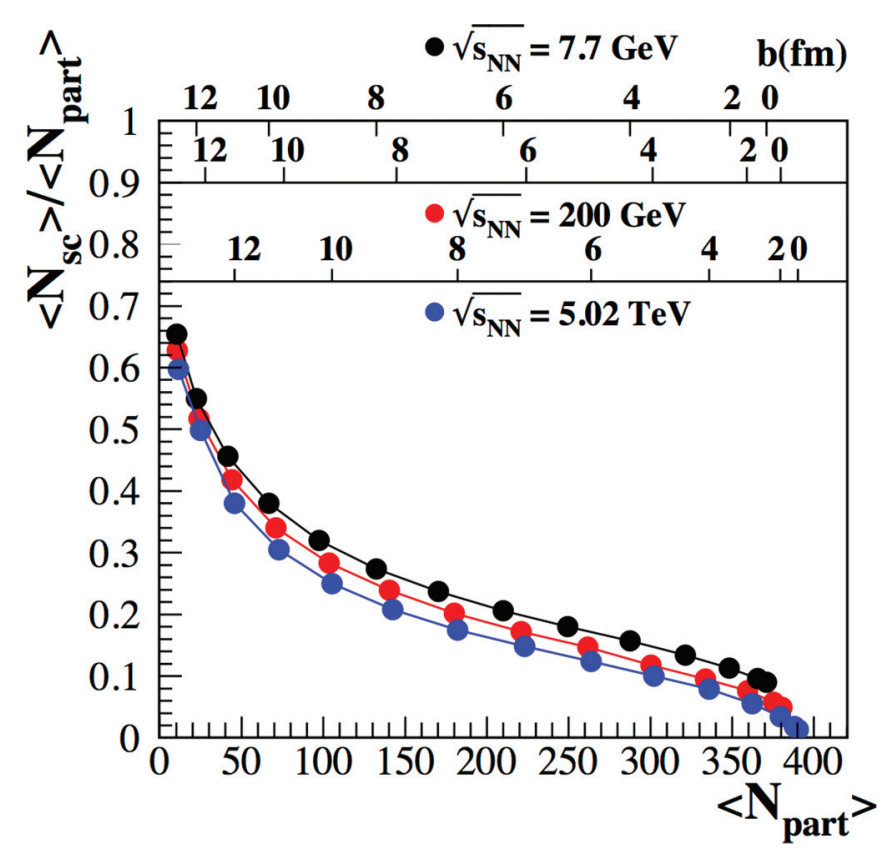

FIG. 3. The percentage of the nucleons suffering a single collision as a function of $\left\langle N_{\text {part }}\right\rangle$ and impact parameter for $\mathrm{Au}-\mathrm{Au}$ collisions at $\sqrt{s_{N N}}=7.7$ and $200 \mathrm{GeV}$ and $\mathrm{Pb}-\mathrm{Pb}$ collisions at $\sqrt{s_{N N}}=5.02 \mathrm{TeV}$. 

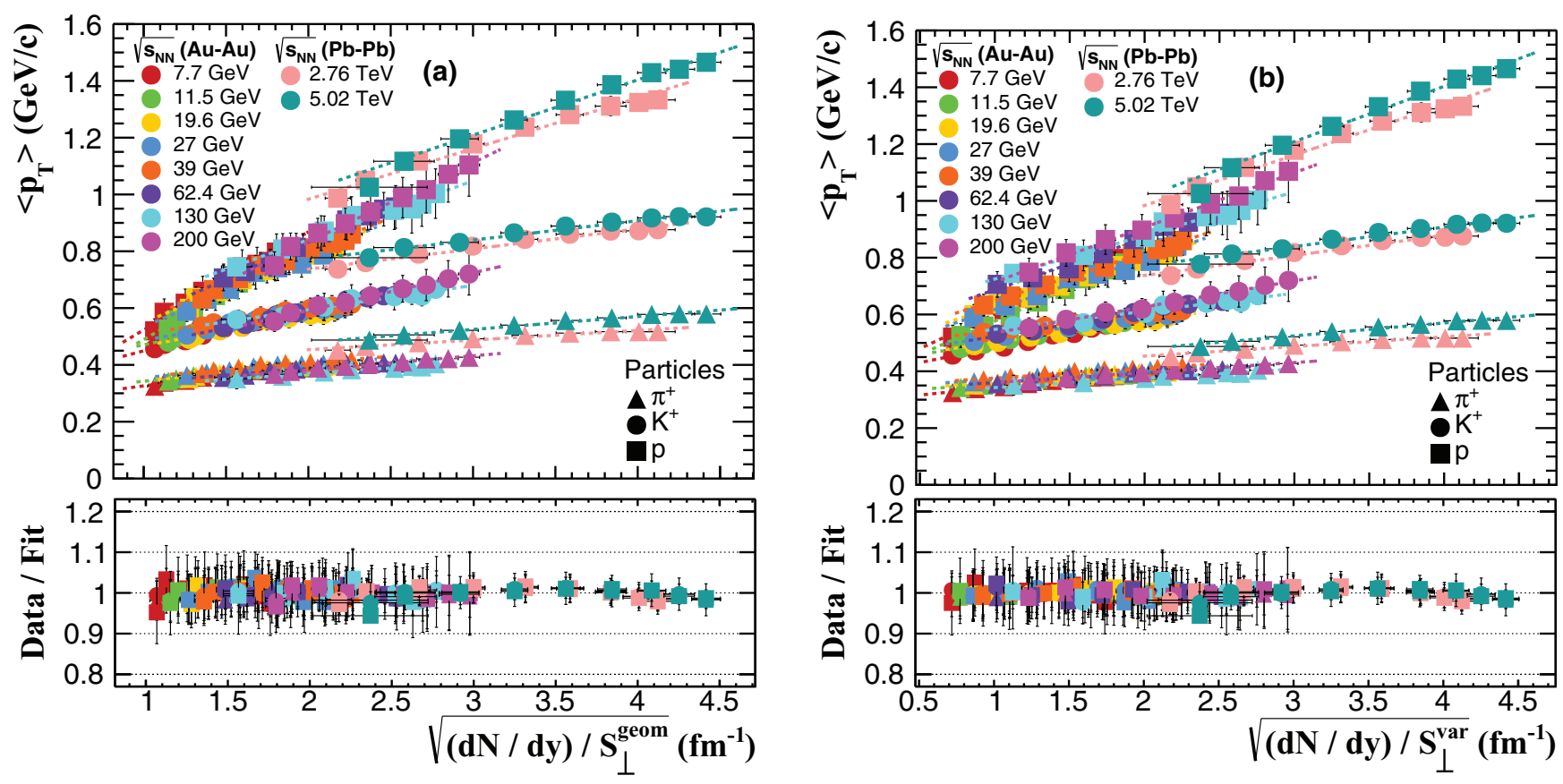

FIG. 4. (a) Top: $\left\langle p_{T}\right\rangle$ of pions, kaons, and protons for all measured energies and centralities at RHIC and LHC reported by the STAR $[34,38]$ and ALICE $[35,40]$ Collaborations, with dashed lines representing the results of the first-order polynomial fit; bottom: the ratio of the data points to the result of the linear fit for each collision energy, as a function of $\sqrt{\frac{d N}{d y} / S_{\perp}^{\text {geom }}}$. (b) Same as (a) but as a function of $\sqrt{\frac{d N}{d y} / S_{\perp}^{\text {var }}}$.

\section{III. $d N / d y$ ESTIMATES}

The total hadron density per unit of rapidity has been estimated based on the published identified charged hadrons densities [34,35,38,40] and hyperons densities [41-47]. For $\sqrt{s_{N N}}=19.6$ and $27 \mathrm{GeV}$ BES energies or some of the centralities, where the hyperon yields were not reported, the corresponding values were obtained by interpolation using the energy and centrality dependence fits.

Because $\Omega^{-}$and $\bar{\Omega}^{+}$yield values for BES were not reported and the extrapolation from higher energies down to BES energies shows a negligible contribution, they were not considered in the produced hadron density estimates. Therefore, we used the following approximations: for the BES energies $\frac{d N}{d y} \simeq \frac{3}{2} \frac{d N}{d y}{ }^{\left(\pi^{+}+\pi^{-}\right)}+2 \frac{d N}{d y}^{\left(K^{+}+K^{-}, p+\bar{p}, \Xi^{-}+\bar{\Xi}^{+}\right)}+$ $\frac{d N}{d y}^{(\Lambda+\bar{\Lambda})}$, from $\sqrt{s_{N N}}=62.4 \mathrm{GeV}$ to $\sqrt{s_{N N}}=200 \mathrm{GeV} \frac{d N}{d y} \simeq$ $\frac{3}{2} \frac{d N}{d y}^{\left(\pi^{+}+\pi^{-}\right)}+2 \frac{d N}{d y}^{\left(K^{+}+K^{-}, p+\bar{p}, \Xi^{-}+\bar{\Xi}^{+}\right)}+\frac{d N}{d y}^{\left(\Lambda+\bar{\Lambda}, \Omega^{-}+\Omega^{-}\right)}$, and for the LHC energies $\frac{d N}{d y} \simeq \frac{3}{2} \frac{d N}{d y}{ }^{\left(\pi^{+}+\pi^{-}\right)}+$ $2 \frac{d N}{d y}^{\left(p+\bar{p}, \Xi^{-}+\bar{\Xi}^{+}\right)}+\frac{d N}{d y}^{\left(K^{+}+K^{-}, K_{S}^{0}+\bar{K}_{S}^{0}, \Lambda+\bar{\Lambda}, \Omega^{-}+\bar{\Omega}^{+}\right)}$. The values are listed in the last column of Table I.

\section{IV. $\sqrt{\frac{d N}{d y} / S_{\perp}}$ DEPENDENCE OF $\left\langle p_{T}\right\rangle$}

As already mentioned in the Introduction, in the local parton-hadron duality approach [8], $\left\langle p_{T}\right\rangle / \sqrt{\frac{d N}{d y} / S_{\perp}}$ is proportional to $\frac{1}{n \sqrt{n}}$, where $n$ is the number of charged hadrons produced via gluon fragmentation $[9,10]$. Therefore, neglecting other effects like collective hydrodynamic expansion and suppression, $\left\langle p_{T}\right\rangle / \sqrt{\frac{d N}{d y} / S_{\perp}}$ is expected to decrease in central collisions and at higher energies. $\left\langle p_{T}\right\rangle$ values for $\mathrm{Au}-\mathrm{Au}$ collisions at $\sqrt{s_{N N}}=7.7,11.5,19.6,27,39 \mathrm{GeV}$ [38] and at $\sqrt{s_{N N}}=62.4,130,200 \mathrm{GeV}$ [34] and for $\mathrm{Pb}-\mathrm{Pb}$ collisions at $\sqrt{s_{N N}}=2.76,5.02 \mathrm{TeV}[35,40]$ for positive pions, kaons, and protons are represented as a function of $\sqrt{\frac{d N}{d y} / S_{\perp}}$ in Fig. 4(a) for $S_{\perp}^{\text {geom }}$ and in Fig. 4(b) for $S_{\perp}^{\text {var }}$. The data points corresponding to each collision energy were fitted with a first-order polynomial function. The trends in the two figures are rather similar and the fit quality, in terms of data/fit ratios, presented in the bottom plots of Fig. 4, is equally good. The fit parameters are listed in Tables II and III for $S_{\perp}=S_{\perp}^{\text {geom }}$ and $S_{\perp}=S_{\perp}^{\mathrm{var}}$, respectively, and represented in Fig. 5 .

The slope value increases from pions to protons. Although the experimental error bars are rather large at the RHIC energies, a systematic decrease of the slopes with the collision energy is evidenced for the $\left\langle p_{T}\right\rangle$ dependence on $\sqrt{\frac{d N}{d y} / S_{\perp}^{\text {geom }}}$ (full symbols). This trend is enhanced going from pions to protons. The offset values are rather similar at the RHIC energies and increase for all the three species at LHC energies. Using $S_{\perp}^{\mathrm{var}}$, Fig. 4(b), the extracted slopes, represented in Fig. 5(a) by open symbols, show a marginal variation as a function of collision energy (dashed lines). The corresponding offsets, represented in Fig. 5(b) by open symbols, within the error bars, are the same for pions and kaons and are systematically larger for protons at RHIC energies compared with the ones corresponding to $S_{\perp}^{\text {geom }}$. We remark that, at LHC energies, the results using $S_{\perp}^{\text {geom }}$ or $S_{\perp}^{\text {var }}$ are the same.

At the LHC energies, in the most central collisions, a saturation trend seems to develop. Natural questions that arise are how much of the observed trends are due to core-corona interplay [48-55] and what does the 
TABLE II. The parameters for the linear fit of the $\left\langle p_{T}\right\rangle$ dependence on $\sqrt{\frac{d N}{d y} / S_{\perp}^{\text {geom }}}$ for pions, kaons, and protons corresponding to the energies mentioned in the first column.

\begin{tabular}{|c|c|c|c|c|c|c|}
\hline$\sqrt{s_{N N}}(\mathrm{GeV})$ & \multicolumn{3}{|c|}{ Slope } & \multicolumn{3}{|c|}{ Offset } \\
\hline 7.7 & $0.08 \pm 0.02$ & $0.15 \pm 0.03$ & $0.35 \pm 0.07$ & $0.25 \pm 0.03$ & $0.30 \pm 0.04$ & $0.18 \pm 0.10$ \\
\hline 11.5 & $0.05 \pm 0.02$ & $0.12 \pm 0.03$ & $0.33 \pm 0.07$ & $0.29 \pm 0.04$ & $0.35 \pm 0.05$ & $0.16 \pm 0.10$ \\
\hline 19.6 & $0.05 \pm 0.02$ & $0.11 \pm 0.04$ & $0.24 \pm 0.05$ & $0.30 \pm 0.04$ & $0.36 \pm 0.06$ & $0.31 \pm 0.08$ \\
\hline 27 & $0.05 \pm 0.02$ & $0.10 \pm 0.03$ & $0.25 \pm 0.05$ & $0.31 \pm 0.04$ & $0.39 \pm 0.06$ & $0.29 \pm 0.08$ \\
\hline 130 & $0.04 \pm 0.02$ & $0.09 \pm 0.02$ & $0.21 \pm 0.05$ & $0.29 \pm 0.04$ & $0.42 \pm 0.05$ & $0.43 \pm 0.10$ \\
\hline 200 & $0.05 \pm 0.02$ & $0.13 \pm 0.04$ & $0.28 \pm 0.06$ & $0.28 \pm 0.04$ & $0.33 \pm 0.09$ & $0.27 \pm 0.13$ \\
\hline 2760 & $0.03 \pm 0.01$ & $0.07 \pm 0.01$ & $0.18 \pm 0.02$ & $0.39 \pm 0.03$ & $0.60 \pm 0.05$ & $0.63 \pm 0.05$ \\
\hline 5020 & $0.05 \pm 0.01$ & $0.07 \pm 0.01$ & $0.19 \pm 0.02$ & $0.39 \pm 0.04$ & $0.64 \pm 0.04$ & $0.63 \pm 0.06$ \\
\hline
\end{tabular}

$\left\langle p_{T}\right\rangle-\sqrt{\frac{d N}{d y} / S_{\perp}}$ correlation for the core look like. Based on the recipe presented in [55], we estimated the $\left\langle p_{T}\right\rangle^{\text {core }}$ for pions, kaons, and protons for $\sqrt{s_{N N}}=200 \mathrm{GeV}, 2.76 \mathrm{TeV}$, and $5.02 \mathrm{TeV}$ :

$$
\left\langle p_{T}\right\rangle_{i}^{\text {cen }}=\frac{f_{\text {core }}\left\langle p_{T}\right\rangle_{i}^{\text {core }} M_{i}^{\text {core }}+\left(1-f_{\text {core }}\right)\left\langle p_{T}\right\rangle_{i}^{p p M B} M_{i}^{p p M B}}{f_{\text {core }} M_{i}^{\text {core }}+\left(1-f_{\text {core }}\right) M_{i}^{p p M B}} .
$$

$\left\langle p_{T}\right\rangle_{i}^{p p M B}$ for $\pi^{+}, K^{+}$, and $p$ in $p p$ minimum bias (MB) collisions at $\sqrt{s_{N N}}=200 \mathrm{GeV}$ were reported by the STAR Collaboration [34] and those at $\sqrt{s_{N N}}=2.76$ and $5.02 \mathrm{TeV}$ were reported by the ALICE Collaboration [56,57]. $(d N / d y)^{\text {core }}$ at the same energies were estimated using

$$
\left(\frac{d N}{d y}\right)_{i}^{\text {cen }}=\left\langle N_{\text {part }}\right\rangle\left[\left(1-f_{\text {core }}\right) M_{i}^{p p M B}+f_{\text {core }} M_{i}^{\text {core }}\right],
$$

where $M_{i}^{p p M B}=\frac{1}{2}(d N / d y)_{i}^{p p M B}$ at the same energy and $M_{i}^{\text {core }}$ is the multiplicity per core participant. $(d N / d y)_{i}^{p p M B}$ for $\pi^{+}, K^{+}$, and $p$ were obtained based on the MB $p_{T}$ spectra reported in $[56,57]$.

In Fig. 6(a), $\left\langle p_{T}\right\rangle$ as a function of $\sqrt{\frac{d N}{d v} / S_{\perp}^{\text {geom }}}$ for pions, kaons, and protons for $\sqrt{s_{N N}}=200 \mathrm{GeV}, 2.76 \mathrm{TeV}$, and 5.02
$\mathrm{TeV}$ is represented. The experimental points for each energy and each species were fitted with linear functions. As already mentioned above, at $\sqrt{s_{N N}}=2.76$ and $5.02 \mathrm{TeV}$ the very last three points, corresponding to the most central collisions, systematically deviate from a linear trend observed at lower centralities and therefore were excluded from the fit. The slopes and the offsets are presented in Table IV. The fit quality can be followed in the bottom plot of Fig. 6(a), where the ratios between the data points and fit results are represented. One can also observe that the last three points at $\sqrt{s_{N N}}=2.76$ and $5.02 \mathrm{TeV}$, corresponding to the most central collisions, deviate from the general trend: the ratio $\left\langle p_{T}\right\rangle / \sqrt{\frac{d N}{d y} / S_{\perp}}$ is decreasing, as expected in Ref. [10].

For $\sqrt{s_{N N}}=200 \mathrm{GeV}, 2.76 \mathrm{TeV}$, and $5.02 \mathrm{TeV}$ we estimated $\left\langle p_{T}\right\rangle^{\text {core }}$ and $\sqrt{\frac{d N}{d y}}{ }^{\text {core }} /\left(S_{\perp}^{\text {geom }}\right)^{\text {core }}$, the results being presented in Fig. 6(b).

The quality of the linear fit, represented in the bottom plot of Fig. 6(b) is equally as good as that for the experimental data, but the slope values presented in Table $\mathrm{V}$ are systematically smaller and the difference between the highest RHIC energy and the LHC energies is reduced. The saturation towards the most central collisions at LHC energies does not change.

TABLE III. The parameters for the linear fit of the $\left\langle p_{T}\right\rangle$ dependence on $\sqrt{\frac{d N}{d y} / S_{\perp}^{\mathrm{var}}}$ for pions, kaons, and protons corresponding to the

\begin{tabular}{|c|c|c|c|c|c|c|}
\hline$\sqrt{s_{N N}}(\mathrm{GeV})$ & \multicolumn{3}{|c|}{ Slope } & \multicolumn{3}{|c|}{ Offset } \\
\hline 7.7 & $0.05 \pm 0.02$ & $0.11 \pm 0.02$ & $0.24 \pm 0.05$ & $0.29 \pm 0.02$ & $0.38 \pm 0.03$ & $0.36 \pm 0.06$ \\
\hline 11.5 & $0.04 \pm 0.02$ & $0.09 \pm 0.02$ & $0.24 \pm 0.04$ & $0.32 \pm 0.02$ & $0.42 \pm 0.03$ & $0.34 \pm 0.06$ \\
\hline 19.6 & $0.04 \pm 0.02$ & $0.08 \pm 0.02$ & $0.17 \pm 0.03$ & $0.33 \pm 0.03$ & $0.42 \pm 0.04$ & $0.46 \pm 0.05$ \\
\hline 27 & $0.03 \pm 0.02$ & $0.07 \pm 0.02$ & $0.18 \pm 0.03$ & $0.34 \pm 0.03$ & $0.45 \pm 0.04$ & $0.44 \pm 0.05$ \\
\hline 130 & $0.03 \pm 0.01$ & $0.06 \pm 0.02$ & $0.15 \pm 0.03$ & $0.31 \pm 0.03$ & $0.48 \pm 0.04$ & $0.57 \pm 0.06$ \\
\hline 200 & $0.04 \pm 0.01$ & $0.09 \pm 0.03$ & $0.19 \pm 0.04$ & $0.33 \pm 0.03$ & $0.44 \pm 0.05$ & $0.52 \pm 0.08$ \\
\hline 2760 & $0.03 \pm 0.01$ & $0.07 \pm 0.01$ & $0.18 \pm 0.02$ & $0.39 \pm 0.03$ & $0.60 \pm 0.04$ & $0.63 \pm 0.05$ \\
\hline 5020 & $0.05 \pm 0.01$ & $0.07 \pm 0.01$ & $0.19 \pm 0.02$ & $0.39 \pm 0.04$ & $0.64 \pm 0.04$ & $0.62 \pm 0.06$ \\
\hline
\end{tabular}
energies mentioned in the first column. 

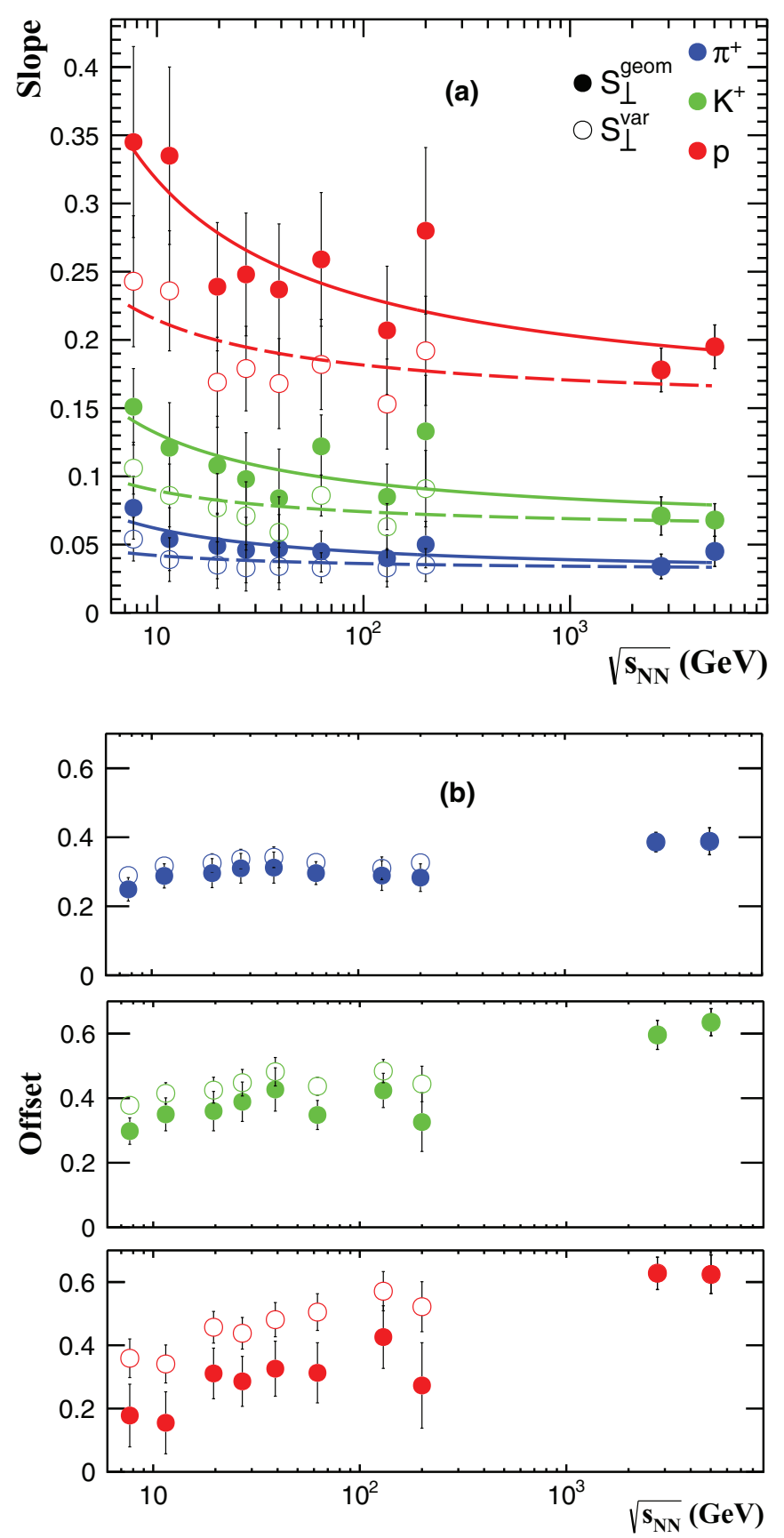

FIG. 5. (a) The slopes of the $\left\langle p_{T}\right\rangle$ dependence on $\sqrt{\frac{d N}{d y} / S_{\perp}^{\text {geom }}}$ (full symbols) and on $\sqrt{\frac{d N}{d y} / S_{\perp}^{\text {var }}}$ (open symbols) for $\pi^{+}$(blue), $K^{+}$(green), and $p$ (red) as a function of $\sqrt{s_{N N}}$. The fit results with the function $a+b /\left(\ln \sqrt{s_{N N}}\right)$ are drawn with full $\left(S_{\perp}^{\text {geom }}\right)$ and dashed $\left(S_{\perp}^{\mathrm{var}}\right)$ lines. (b) The corresponding offsets.

\section{V. $\left\langle p_{T}\right\rangle$ PARTICLE MASS DEPENDENCE AS A FUNCTION OF $\sqrt{\frac{d N}{d y} / S_{\perp}^{\text {geom }}}$}

The $\left\langle p_{T}\right\rangle$ dependence on the mass of pions, kaons, and protons at different collision centralities, except for the most peripheral ones, is linear. Therefore, linear fits of the $\left\langle p_{T}\right\rangle$ particle mass dependence, corresponding to each centrality and energy considered in the paper, were performed. The extracted
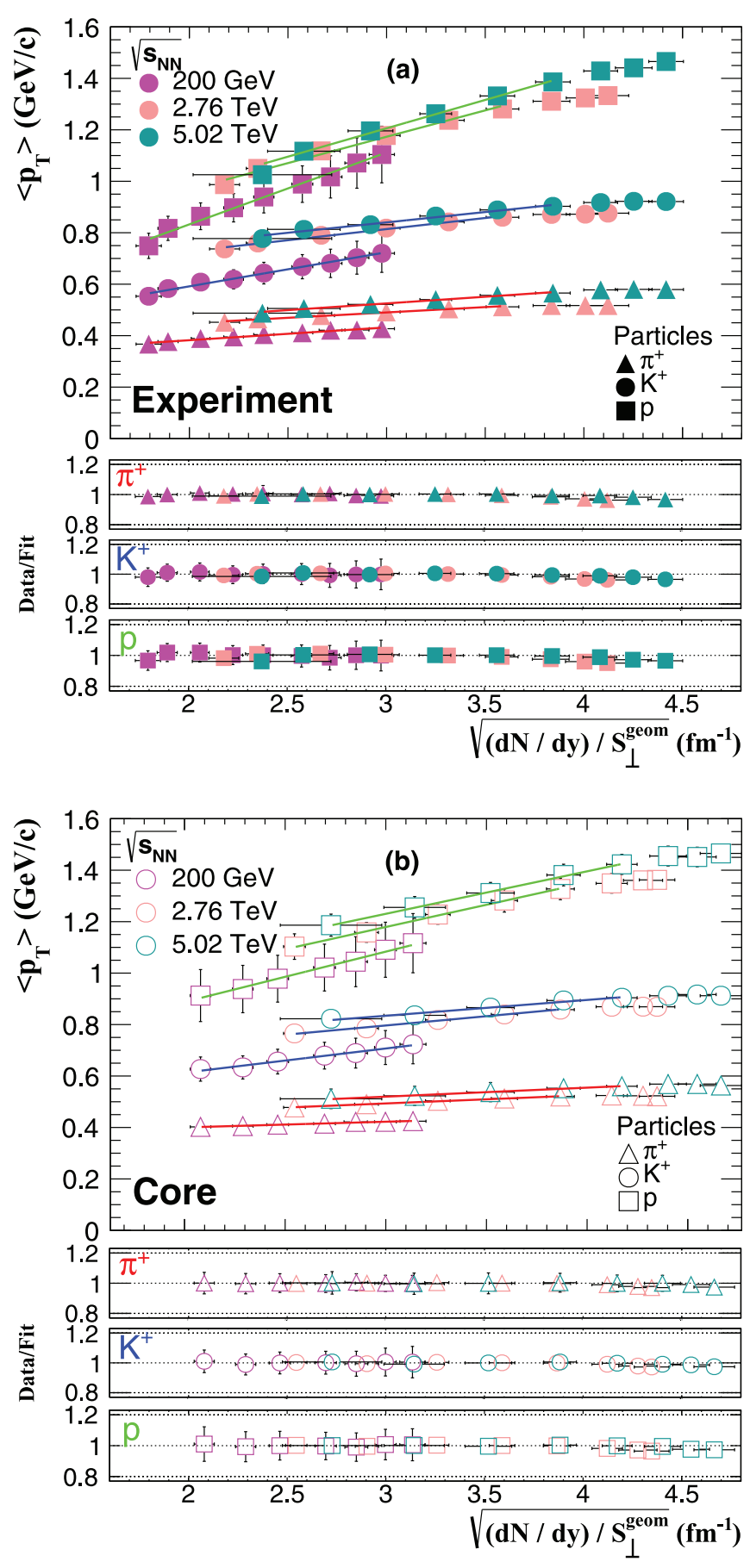

FIG. 6. $\left\langle p_{T}\right\rangle$ as a function of $\sqrt{\frac{d N}{d y} / S_{\perp}^{\text {geom }}}$ for identified charged hadrons for $\sqrt{s_{N N}}=200 \mathrm{GeV}, 2.76 \mathrm{TeV}$, and $5.02 \mathrm{TeV}$. The full lines represent the results of the first-order polynomial fit. (a) Top: experimental results; bottom: data/fit ratio. (b) Top: estimated core contribution; bottom: data/fit ratio.

fit parameters as a function of $\sqrt{\frac{d N}{d y} / S_{\perp}^{\text {geom }}}$ are shown in Fig. 7 (slope) and Fig. 8 (offset). In Fig. 7 the slopes are fitted with the following expression:

$$
\operatorname{Slope}_{\left\langle p_{T}\right\rangle=f(\text { mass })}=\alpha+\beta\left(\sqrt{\frac{d N}{d y} / S_{\perp}^{\text {geom }}}\right)^{\gamma},
$$


TABLE IV. The parameters of the linear fit of $\left\langle p_{T}\right\rangle$ as a function of $\sqrt{\left(\frac{d N}{d y}\right) / S_{\perp}^{\text {geom }}}$ for pions, kaons, and protons corresponding to $\sqrt{s_{N N}}=$ $200 \mathrm{GeV}, 2.76 \mathrm{TeV}$, and $5.02 \mathrm{TeV}$ collision energies. The very last three points at $\sqrt{s_{N N}}=2.76$ and $5.02 \mathrm{TeV}$ were not included in the fit.

\begin{tabular}{|c|c|c|c|c|c|c|}
\hline$\sqrt{s_{N N}}(\mathrm{GeV})$ & $\pi^{+}$ & $K^{+}$ & $\mathrm{p}$ & $\pi^{+}$ & $K^{+}$ & $\mathrm{p}$ \\
\hline 2760 & $0.04 \pm 0.01$ & $0.09 \pm 0.02$ & $0.20 \pm 0.03$ & $0.37 \pm 0.04$ & $0.56 \pm 0.07$ & $0.56 \pm 0.08$ \\
\hline 5020 & $0.05 \pm 0.02$ & $0.08 \pm 0.02$ & $0.22 \pm 0.03$ & $0.37 \pm 0.06$ & $0.60 \pm 0.07$ & $0.54 \pm 0.10$ \\
\hline
\end{tabular}

The slopes for particles [Fig. 7(a)] and antiparticles [Fig. 7(b)] evidence a $\sqrt{\frac{d N}{d y} / S_{\perp}^{\text {geom }}}$ dependence which closely follows a trend given by Eq. (4) (dashed lines). The values of the fit parameters are listed in Fig. 7. The fit quality is represented in the bottom plots of Figs. 7(a) and 7(b) in terms of data/fit. Besides the points corresponding to the most central collisions at $\sqrt{s_{N N}}=19.6,27$, and $39 \mathrm{GeV}$, which deviate from the fit by $\sim 10 \%-15 \%$, the bulk of data nicely cluster around the fit curve, well within the error bars. In Fig. 8, although the error bars are rather large, a systematic increase of the offsets as a function of $\sqrt{\frac{d N}{d y} / S_{\perp}^{\text {geom }}}$ is evidenced at BES energies and at $\sqrt{s_{N N}}=62.4 \mathrm{GeV}$, reaching a plateau above $1.7 \mathrm{fm}^{-1}$. This trend is much reduced starting from $\sqrt{s_{N N}}=130 \mathrm{GeV}$. Therefore, we considered only offsets above $1.7 \mathrm{fm}^{-1}$ and found their average values for different $\sqrt{s_{N N}}$. The results are presented in Fig. 9.

\section{VI. $\sqrt{\frac{d N}{d y} / S_{\perp}^{\text {geom }}}$ DEPENDENCE OF BOLTZMANN-GIBBS BLAST WAVE FIT PARAMETERS}

The $p_{T}$ spectra for identified charged hadrons were fitted $[34,35,38,40,58]$ using the BGBW expression inspired by hydrodynamic models [59]:

$$
\begin{aligned}
E \frac{d^{3} N}{d p^{3}} \sim & \int_{0}^{R} m_{T} K_{1}\left(m_{T} \cosh \rho / T_{\text {kin }}^{f o}\right) \\
& \times I_{0}\left(p_{T} \sinh \rho / T_{\text {kin }}^{f o}\right) r d r
\end{aligned}
$$

where $m_{T}=\sqrt{m^{2}+p_{T}^{2}}, \quad \beta_{T}(r)=\beta_{s}\left(\frac{r}{R}\right)^{n}, \quad \rho=\tanh ^{-1} \beta_{T}$. $T_{\text {kin }}^{f o}$ is the kinetic freeze-out temperature and $n$ defines the expansion profile. A compilation of all results in terms of the $\left\langle\beta_{T}\right\rangle$ dependence on $\sqrt{\frac{d N}{d y} / S_{\perp}^{\mathrm{geom}}}$ is presented in Fig. 10. We should mention that for the BES energies [38] the BGBW fits were performed simultaneously on particles' and antiparticles' $p_{T}$ spectra, although they do not present the same trends in many respects. Therefore, in Fig. 10, the $\left\langle\beta_{T}\right\rangle$ for antiparticles for some energies and centralities, where the azimuthal dependent BGBW fits were published [60,61], are represented by open symblols. One can observe that, with increasing collision energy, the values of $\left\langle\beta_{T}\right\rangle$ for antiparticles converge towards the values obtained from a simultaneous fit of particles' and antiparticles' $p_{T}$ spectra [34,38]. However, the $\left\langle\beta_{T}\right\rangle$ values reported in the literature scale rather nicely as a function of $\sqrt{\frac{d N}{d y} / S_{\perp}^{\mathrm{geom}}}$, and a fourth-order polynomial function fits them well. The fit quality can be followed in the bottom plot of Fig. 10. Within the experimental error bars, all data follow the fit result, except the points corresponding to the peripheral collisions at the lowest BES energies. The fit parameters are included in the figure. The same representation, in which the data corresponding to $\sqrt{s_{N N}}=7.7,11.5$, and $62.4 \mathrm{GeV}$ are excluded, can be followed in Fig. 11. For the remaining energies, from $\sqrt{s_{N N}}=19.6 \mathrm{GeV}$ to $5.02 \mathrm{TeV}$ a much better scaling is observed. The dynamics in $\left\langle\beta_{T}\right\rangle$ as a function of $\sqrt{\frac{d N}{d y} / S_{\perp}^{\text {geom }}}$ for different collision energies can be more easily followed in Fig. 12, where the ratio between $\left\langle\beta_{T}\right\rangle$ at a given centrality relative to $\left\langle\beta_{T}\right\rangle$ in the most peripheral collisions, $70 \%-80 \%(58 \%-85 \%$ for $130 \mathrm{GeV}),\left\langle\beta_{T}\right\rangle /\left\langle\beta_{T}^{\text {Peripheral }}\right\rangle$, is plotted as a function of $\sqrt{\frac{d N}{d y} / S_{\perp}^{\text {geom }}}$ for all energies.

In Fig. 13 the $T_{\text {kin }}^{f o}$ and $n$ parameters and their dependence on $\sqrt{\frac{d N}{d y} / S_{\perp}^{\text {geom }}}$ are presented. A close to linear dependence with a negative slope is observed in Fig. 13(a), for $T_{\text {kin }}^{f o}$ at RHIC energies. Within the error bars, it is rather difficult to conclude on some collision energy dependence of $T_{\text {kin }}^{f o}$ for a given value of the geometrical variable. On the other hand, a significant shift of about $20 \mathrm{MeV}$ in $T_{\text {kin }}^{\text {fo }}$ fit parameter towards larger values is evidenced for a given $\sqrt{\frac{d N}{d y} / S_{\perp}^{\text {geom }}}$ at LHC energies relative to the RHIC energies. Similar shifts were mentioned in the previous sections for $\left\langle p_{T}\right\rangle$ and the offsets of $\left\langle p_{T}\right\rangle$ as a function of mass. Such a shift is also evidenced in the $T_{\text {kin }}^{\text {fo }}$ versus $\left\langle\beta_{T}\right\rangle$ representation in Fig. 14 where the fit

TABLE V. The parameters of the linear fit of $\left\langle p_{T}\right\rangle$ as a function of $\sqrt{\left(\frac{d N}{d y}\right)^{\text {core }} /\left(S_{\perp}^{\text {geom }}\right)^{\text {core }}}$ for pions, kaons, and protons corresponding to $\sqrt{s_{N N}}=200 \mathrm{GeV}, 2.76 \mathrm{TeV}$, and $5.02 \mathrm{TeV}$ collision energies. For $\sqrt{s_{N N}}=2.76$ and $5.02 \mathrm{TeV}$, the last three centralities, where a levelling off

\begin{tabular}{|c|c|c|c|c|c|c|}
\hline$\sqrt{s_{N N}}(\mathrm{GeV})$ & \multicolumn{3}{|c|}{ Slope } & \multicolumn{3}{|c|}{ Offset } \\
\hline 2760 & $0.03 \pm 0.02$ & $0.07 \pm 0.03$ & $0.17 \pm 0.04$ & $0.40 \pm 0.06$ & $0.58 \pm 0.10$ & $0.66 \pm 0.14$ \\
\hline 5020 & $0.03 \pm 0.03$ & $0.06 \pm 0.02$ & $0.17 \pm 0.04$ & $0.41 \pm 0.11$ & $0.65 \pm 0.08$ & $0.73 \pm 0.16$ \\
\hline
\end{tabular}
is evidenced, were not included in the fit. 

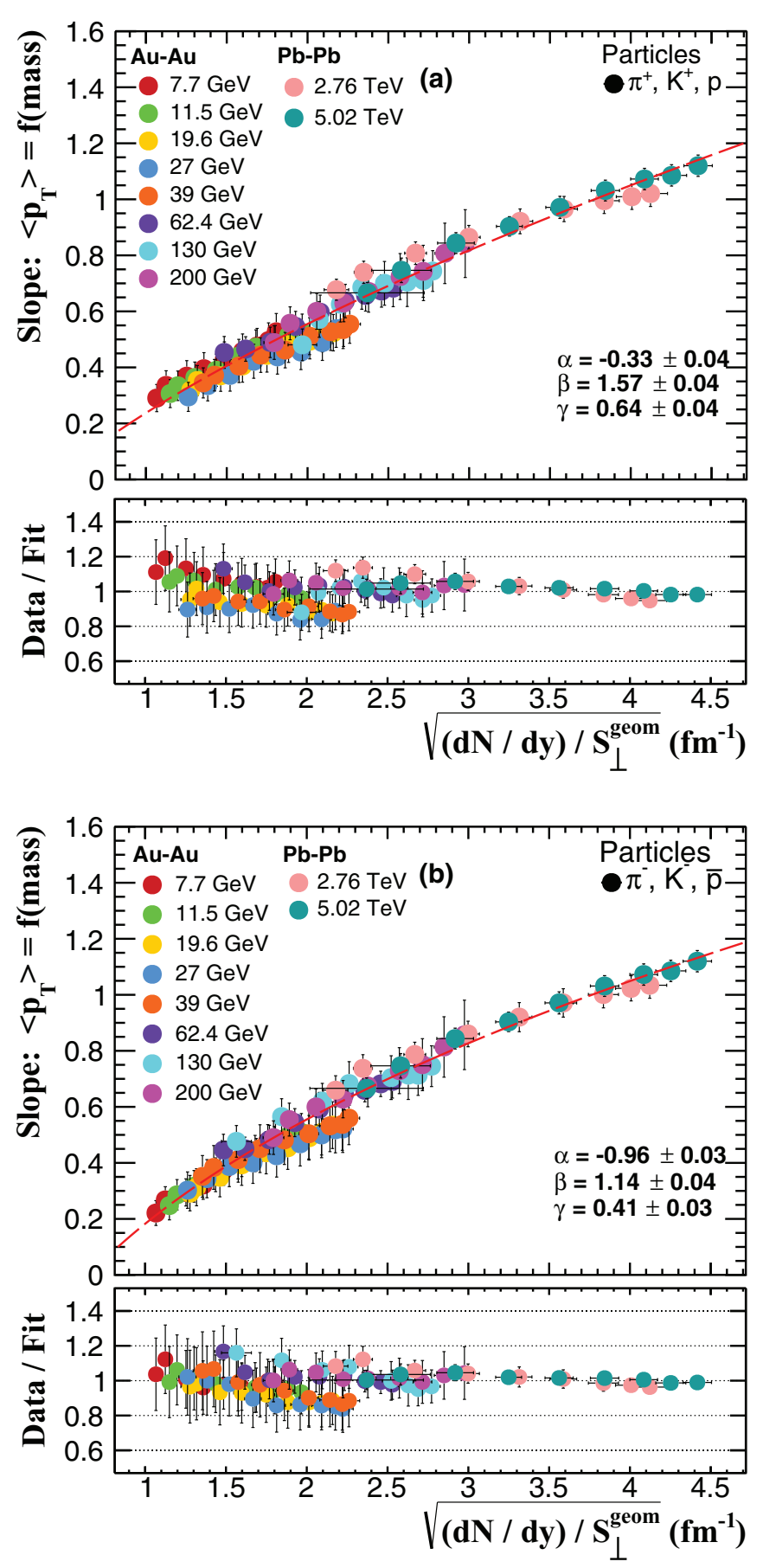

FIG. 7. The slopes from the linear fit of $\left\langle p_{T}\right\rangle$ versus particle mass as a function of $\sqrt{\frac{d N}{d y} / S_{\perp}^{\mathrm{geom}}}$, for each centrality and energy, for (a) $\pi^{+}$, $K^{+}, p$ and (b) $\pi^{-}, K^{-}, \bar{p}$. The continuous red line is the result of the fit with the function from Eq. (4). The data/fit ratios are represented in the bottom plots of each of the two figures.

parameters reported in Ref. [34,35,38,40,58] are used. As far as the $n$ dependence on $\sqrt{\frac{d N}{d y}} / S_{\perp}^{\text {geom }}$ is concerned, Fig. 13(b), the values for BES energies are rather scattered and those corresponding to 62.4 and $200 \mathrm{GeV}$ show an opposite trend to what is observed at LHC. Usually, the flow profile changes from a shell type expansion with large $n$ values, towards $n=1$ (Hubble type) with increasing centrality. Values even smaller
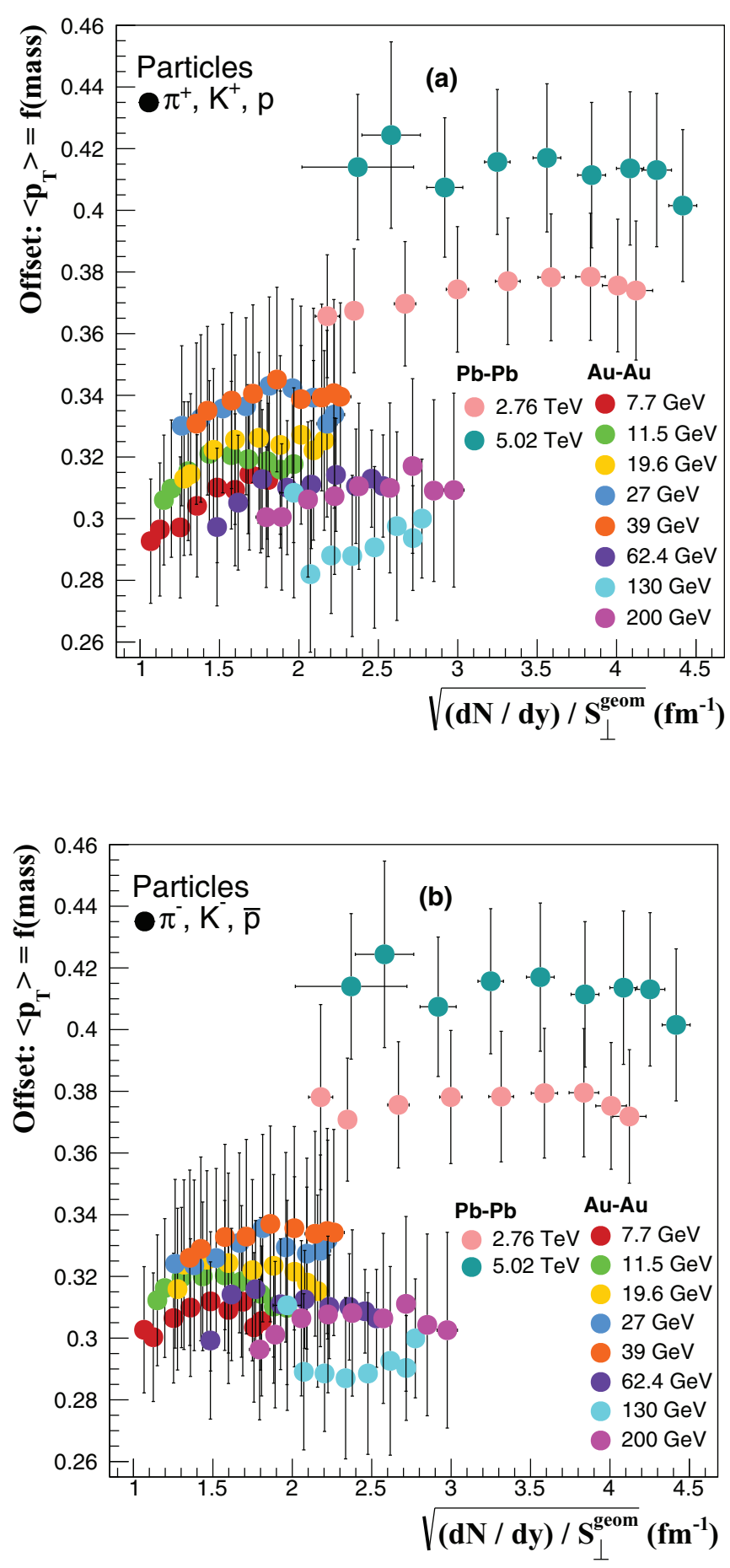

FIG. 8. The offsets from the linear fits of $\left\langle p_{T}\right\rangle$ versus particle mass dependence as a function of $\sqrt{\frac{d N}{d y} / S_{\perp}^{\text {geom }}}$ : (a) particles, (b) antiparticles.

than 1 are obtained for very central collisions. It is worth mentioning that for a consistent interpretation, the fits of the $p_{T}$ spectra using the BGBW expression have to be done at all energies on the same $p_{T}$ range for a given species. The range has to be chosen such to reduce as much as possible the influence of processes other than collective expansion on the extracted fit parameters. Therefore, the lower limit of the fit range for pions has to be chosen such that the contribution 


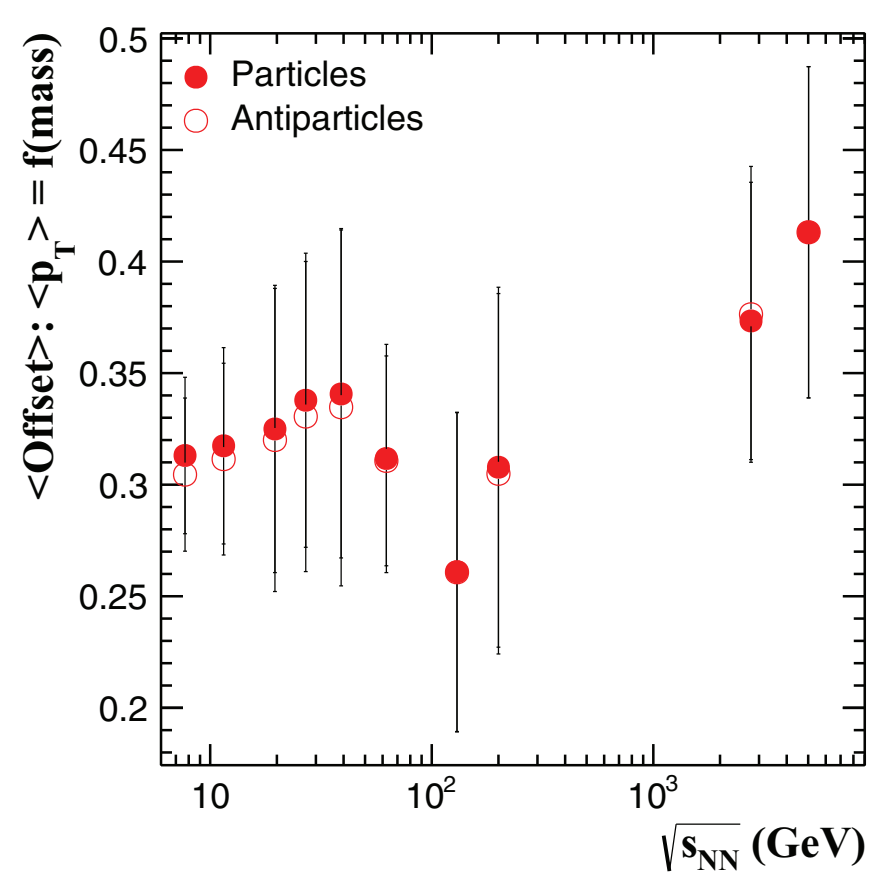

FIG. 9. The average values of the offsets of the $\left\langle p_{T}\right\rangle$ mass dependence for $\sqrt{\frac{d N}{d y} / S_{\perp}^{\text {geom }}} \geqslant 1.7 \mathrm{fm}^{-1}$ as a function of $\sqrt{s_{N N}}$. Full symbols: particles; open symbols: antiparticles.

coming from resonance decays is reduced, while the upper fit ranges for all species have to be optimized in order to be influenced as little as possible by the suppression effects. Last but not least, the influence of the corona contribution on the fit parameters has to be carefully considered.

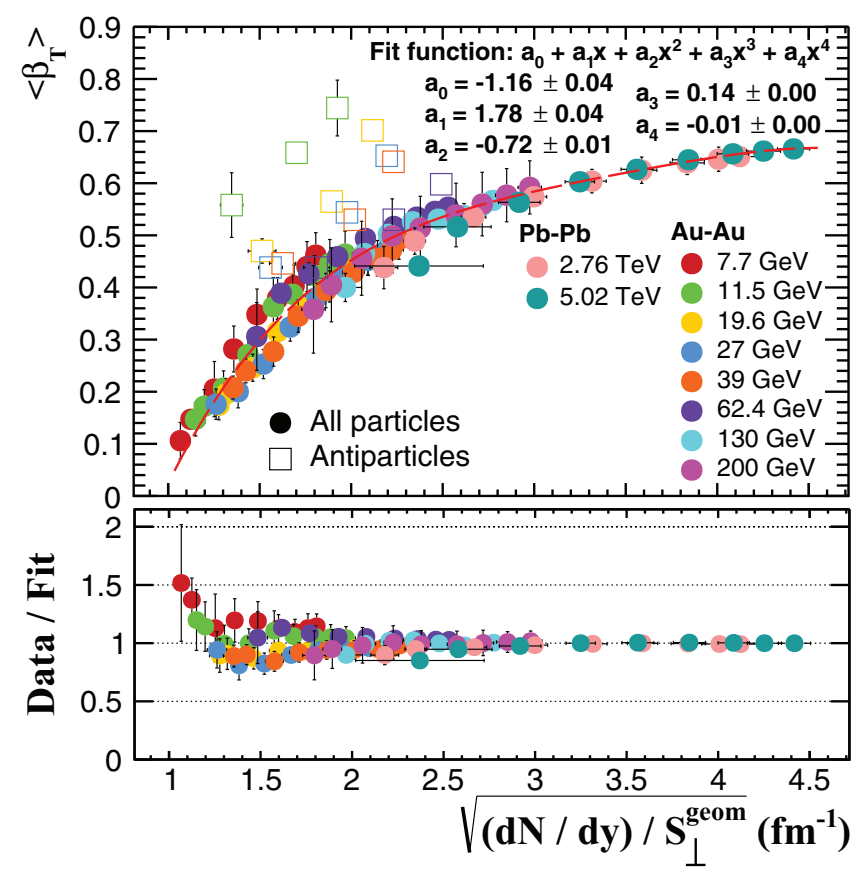

FIG. 10. Top: the BGBW fit parameter $\left\langle\beta_{T}\right\rangle$ as a function of $\sqrt{\frac{d N}{d y} / S_{\perp}^{\mathrm{geom}}}$; the dashed red line corresponds to the result of the fit using a fourth-order polynomial function. Bottom: the data/fit ratio.

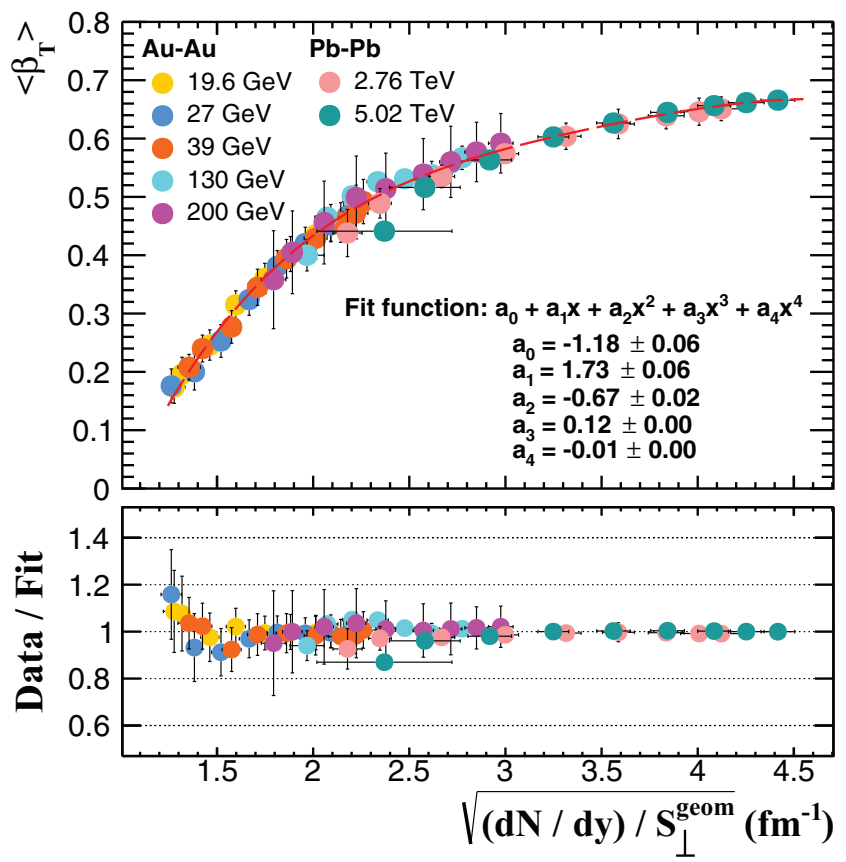

FIG. 11. The BGBW fit parameter $\left\langle\beta_{T}\right\rangle$ as a function of $\sqrt{\frac{d N}{d y} / S_{\perp}^{\mathrm{geom}}}$ excluding $\sqrt{s_{N N}}=7.7,11.5$, and $62.4 \mathrm{GeV}$ collision energies.

\section{COMPARISON BETWEEN $p p$ AND Pb-Pb SYSTEMS AT LHC ENERGIES}

Similarities between $p p$ and $\mathrm{Pb}-\mathrm{Pb}$ in terms of the behavior of different observables, like the $\left(\left\langle\beta_{T}\right\rangle-T_{\text {kin }}^{f o}\right)$ correlation as a function of charged particle multiplicity [15] and nearside long-range pseudorapidity correlations at large charged particle multiplicities [11], were evidenced at LHC energies.

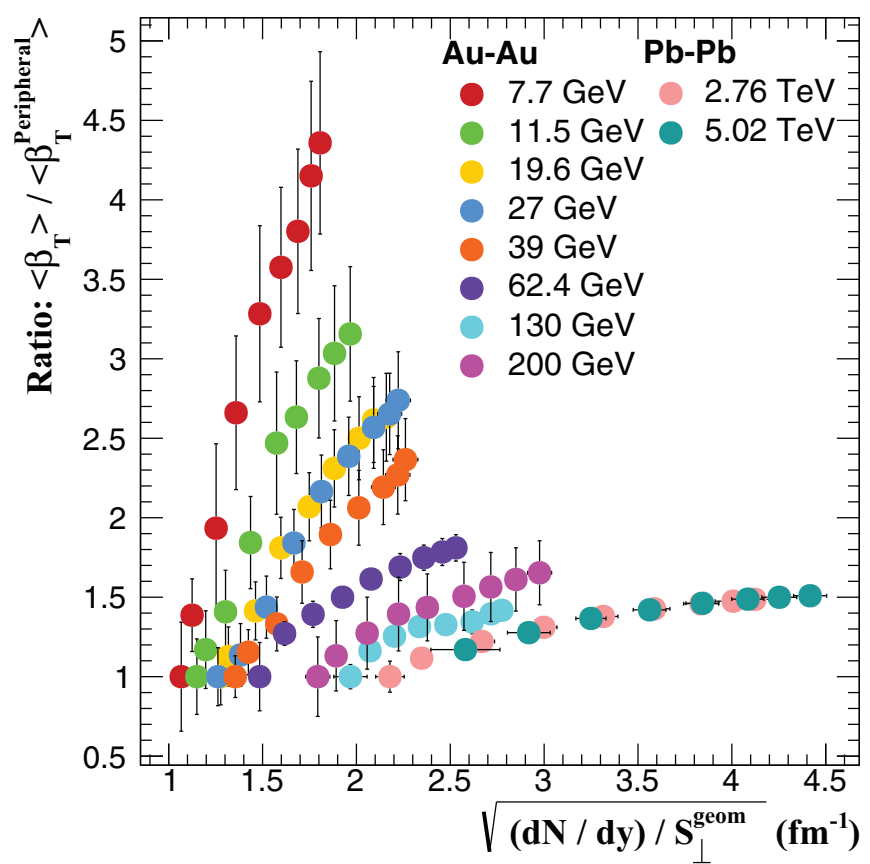

FIG. 12. $\left\langle\beta_{T}\right\rangle /\left\langle\beta_{T}^{\text {Peripheral }}\right\rangle$ as a function of $\sqrt{\frac{d N}{d y} / S_{\perp}^{\text {geom }}} .\left\langle\beta_{T}\right\rangle$ fit parameters were reported in Refs. [34,35,38,40]. 

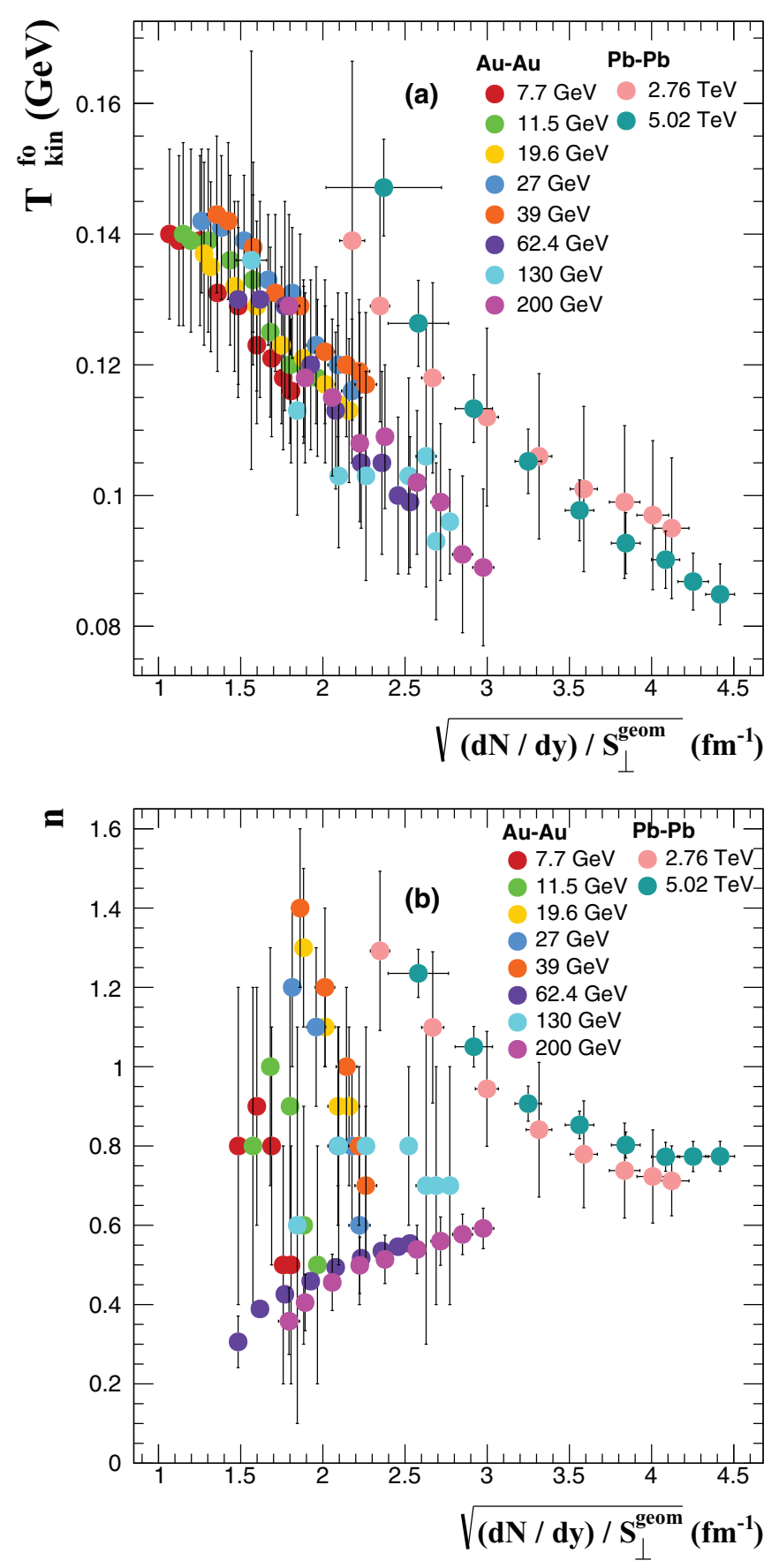

FIG. 13. (a) $T_{\text {kin }}^{f o}$ and (b) $n$ parameters as a function of $\sqrt{\frac{d N}{d y} / S_{\perp}^{\text {geom }}}$. The fit parameters were taken from $[34,35,38,40,58]$.

The extent to which the similarity between $p p$ and $\mathrm{Pb}-\mathrm{Pb}$ is also evidenced in the behavior of the observables described in the

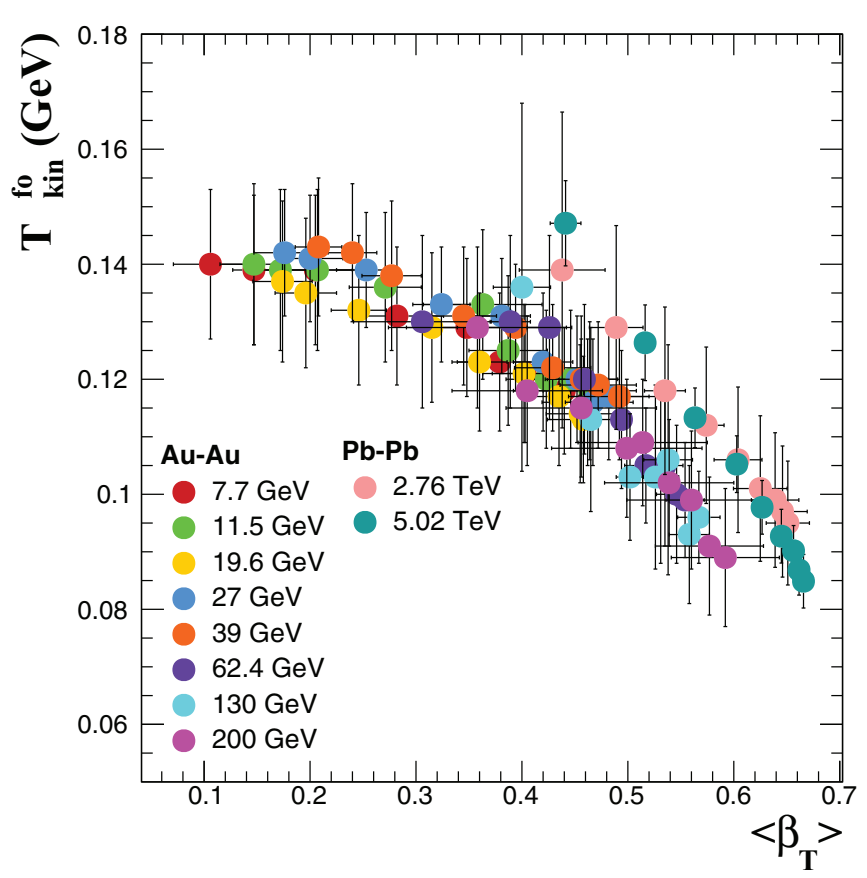

FIG. 14. The BGBW fit parameters $T_{\text {kin }}^{f o}$ versus $\left\langle\beta_{T}\right\rangle$ reported in $[34,35,38,40,58]$.

previous sections as a function of the saturation momentum, i.e., $\sqrt{\frac{d N}{d y} / S_{\perp}}$, is further investigated. For this comparison we used the results of the ALICE Collaboration for $p_{T}$ spectra of identified light flavor charged hadrons as a function of charged particle multiplicity at mid-rapidity as well as the results of their fits with the BGBW expression given by Eq. (5) [15]. The hadron density per unit of rapidity for the mid-central charged particle multiplicity was estimated by extrapolating the results reported by the ALICE Collaboration in Ref. [17]. The $\left\langle p_{T}\right\rangle$ values were estimated based on the $p_{T}$ spectra from [15] extrapolated in the unmeasured regions using fits of the measured spectra with the expression from [62]:

$$
\frac{d \sigma}{p_{T} d p_{T}}=A_{e} \exp \left(-E_{T}^{\mathrm{kin}} / T_{e}\right)+\frac{A}{\left(1+\frac{p_{T}^{2}}{T^{2} n}\right)^{n}} .
$$

The overlapping area for $p p$ collisions, $S_{\perp}^{p p}=\pi R_{p p}^{2}$, is calculated using the estimates of the maximal radius for which the energy density of the Yang-Mill fields is larger than $\varepsilon=$ $\alpha \Lambda_{Q C D}^{4}(\alpha \in[1,10])$ within the IP-Glasma initial state model $[63,64]$. Within the present knowledge of QCD, $\alpha$ cannot be precisely estimated. The $r_{\max }$ values used in Ref. [63] for $\alpha=1$ were fitted in Ref. [65] with the following expression:

$$
f_{p p}= \begin{cases}0.387+0.0335 x+0.274 x^{2}-0.0542 x^{3} & \text { if } x<3.4 \\ 1.538 & \text { if } x \geqslant 3.4\end{cases}
$$

Using the same recipe we fitted the $r_{\max }$ values from Ref. [63] for $\alpha=10$ with the following expression:

$$
f_{p p}= \begin{cases}-0.018+0.3976 x+0.095 x^{2}-0.028 x^{3} & \text { if } x<3.4 \\ 1.17 & \text { if } x \geqslant 3.4\end{cases}
$$


TABLE VI. The hadron density per unit of rapidity and transverse overlapping areas for $\alpha=1$ and $\alpha=10$ for $p p$ collisions at $\sqrt{s}=7$ $\mathrm{TeV}$.

\begin{tabular}{lccc}
\hline \hline \multirow{2}{*}{$\begin{array}{l}\text { s }(\mathrm{TeV}) \\
(p p)\end{array}$} & $d N / d y$ & \multicolumn{2}{c}{$S_{\perp}\left(\mathrm{fm}^{2}\right)$} \\
\cline { 3 - 4 } 7 & & $7.43 \pm 0.48$ & $4.30 \pm 0.36$ \\
& $82.1 \pm 2.8$ & $7.43 \pm 0.41$ & $4.30 \pm 0.31$ \\
& $70.2 \pm 2.2$ & $7.43 \pm 0.35$ & $4.30 \pm 0.27$ \\
& $59.4 \pm 1.7$ & $7.43 \pm 0.30$ & $4.30 \pm 0.23$ \\
& $48.8 \pm 1.3$ & $7.39 \pm 0.02$ & $4.20 \pm 0.02$ \\
& $37.3 \pm 0.9$ & $6.89 \pm 0.05$ & $3.80 \pm 0.03$ \\
& $26.8 \pm 0.6$ & $5.94 \pm 0.06$ & $3.16 \pm 0.04$ \\
& $18.2 \pm 0.4$ & $4.58 \pm 0.06$ & $2.29 \pm 0.04$ \\
\hline \hline
\end{tabular}

where $x=\left(d N_{g} / d y\right)^{1 / 3}$. The hadron density per unit of rapidity, estimated based on the approximation $\frac{d N}{d y} \simeq$ $\frac{3}{2} \frac{d N}{d y}{ }^{\left(\pi^{+}+\pi^{-}\right)}+2 \frac{d N}{d y}{ }^{\left(p+\bar{p}, \Xi^{-}+\bar{\Xi}^{+}, K_{S}^{0}\right)}+\frac{d N}{d y}^{\left(K^{+}+K^{-}, \Lambda+\bar{\Lambda}, \Omega^{-}+\bar{\Omega}^{+}\right)}$, and the corresponding overlapping areas for $\alpha=1$ and $\alpha=10$ values are listed in Table VI. The gluon density per unit of rapidity was approximated by $d N_{g} / d y \approx d N / d y$. The comparison between the $\left\langle p_{T}\right\rangle$ dependence on the square root of the hadron density per unit of rapidity and unit of interaction area for the $p p$ collisions at $\sqrt{s}=7 \mathrm{TeV}$ and $\mathrm{Pb}-\mathrm{Pb}$ collisions at $\sqrt{s_{N N}}=2.76$ and $5.02 \mathrm{TeV}$, based on the results obtained by the ALICE Collaboration [15,35,40,58], is presented in Fig. 15. As one can see, the general trend for all the three species is very similar in $p p$ and $\mathrm{Pb}-\mathrm{Pb}$ collisions.

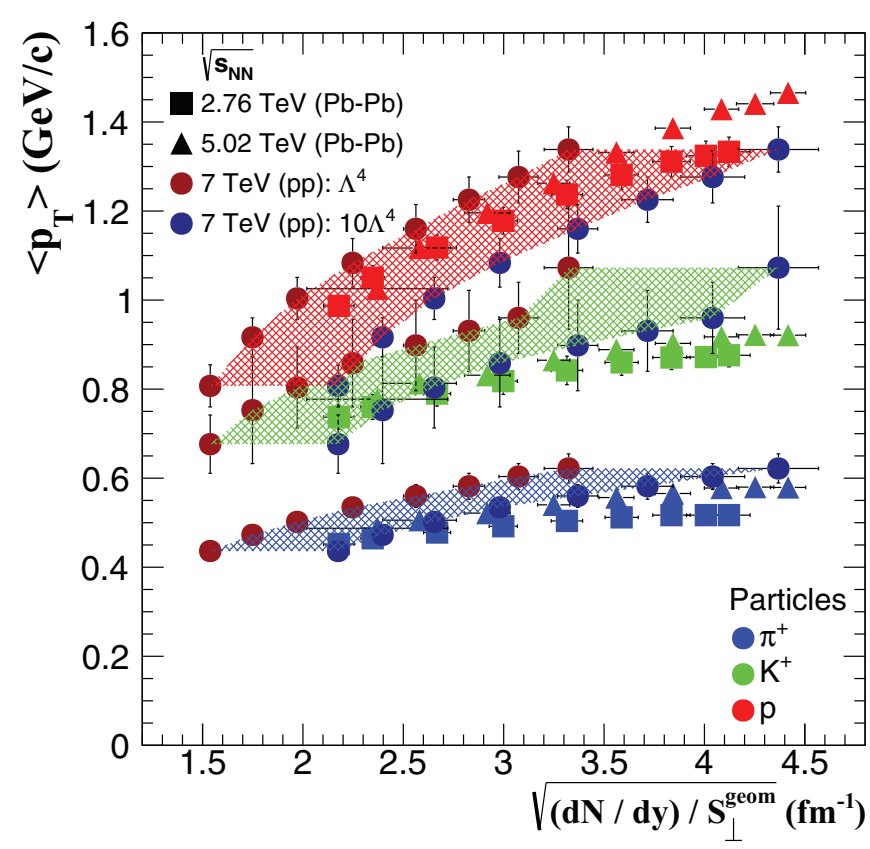

FIG. 15. $\left\langle p_{T}\right\rangle$ for identified charged hadrons for $p p$ collisions at $\sqrt{s}=7 \mathrm{TeV}$ [15] (dark red symbols: $\alpha=1$; dark blue symbols: $\alpha=10$ ) and $\mathrm{Pb}-\mathrm{Pb}$ collisions at $\sqrt{s_{N N}}=2.76$ and $5.02 \mathrm{TeV}[35,40]$. The blue, green, and red shaded areas represent the uncertainty in the overlapping surface area estimates for $p p$ collisions; see the text.

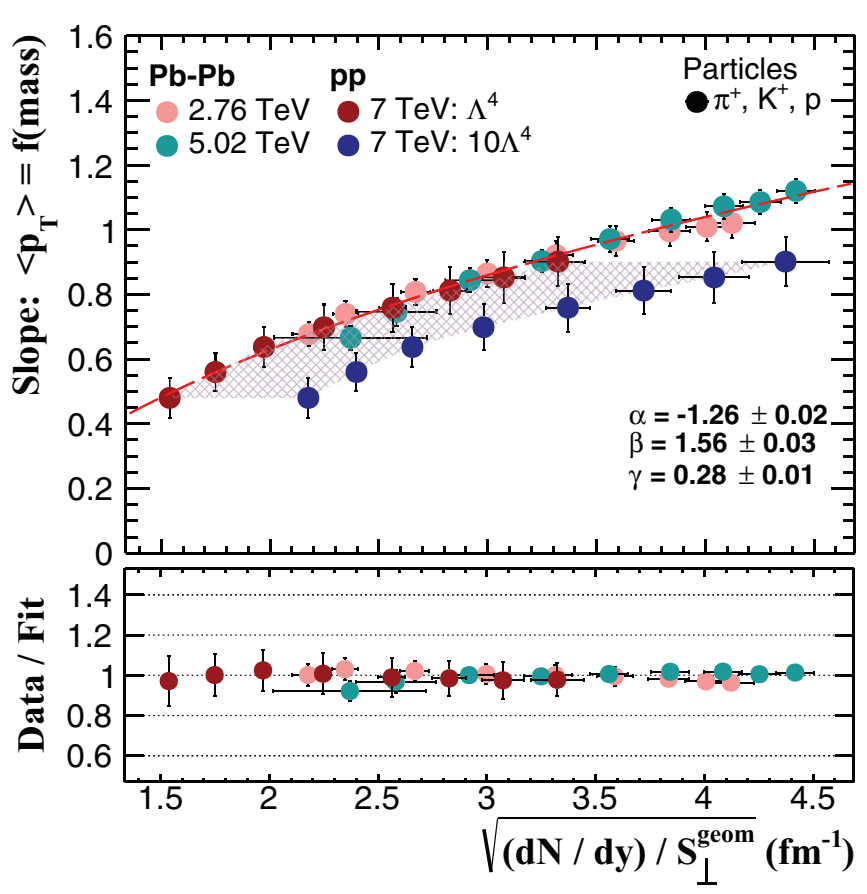

FIG. 16. The slopes of the $\left\langle p_{T}\right\rangle$ particle mass dependence as a function of $\sqrt{\frac{d N}{d y} / S_{\perp}^{\text {geom }}}$ for $p p$ at $\sqrt{s}=7 \mathrm{TeV}$ (red symbols: $\alpha=1$; blue symbols: $\alpha=10$ ) and Pb-Pb at $\sqrt{s_{N N}}=2.76$ and $5.02 \mathrm{TeV}$.

The differences could have several origins, i.e., the difference in the collision energies, a systematic larger $\left\langle p_{T}\right\rangle$ for kaons in $p p$ relative to $\mathrm{Pb}-\mathrm{Pb}$, uncertainty in estimating the value of $\alpha$, the large inhomogeneity of the initial state with a direct consequence on the $S_{\perp}$ estimate, and last but not least the buildup of collective expansion in the hadronic phase and suppression effects taking place in the $\mathrm{Pb}-\mathrm{Pb}$ case and not yet evidenced in $p p$ collisions. The comparison between the two systems in terms of the slopes of the $\left\langle p_{T}\right\rangle$ particle mass dependence as a function of $\sqrt{\frac{d N}{d y} / S_{\perp}^{\text {geom }}}$ is presented in Fig. 16. A very good scaling is found using $\alpha=1$ for $p p$ collisions. The same value of $\alpha$ was used in Refs. [65,66]. These results seem to support the assumption that the global properties of the hadron production are determined by the properties of flux tubes of $\sim 1 / \sqrt{\frac{d N}{d y} / S_{\perp}}$ size and are very little influenced by the size of the colliding system $[18,65,67]$. A similar behavior was evidenced at the baryonic level at much lower energies, where the main features of the dynamic evolution of the fireball are determined by the initial baryon density profile and temperature and not too much by its size [68]. As is well known, the LPHD approach neglects all collective effects. However, a comparison between $p p$ and $\mathrm{Pb}-\mathrm{Pb}$ collisions in terms of $\left\langle\beta_{T}\right\rangle$, one of the BGBW fit parameters interpreted as the average transverse flow velocity, could be rather interesting. $\left\langle\beta_{T}\right\rangle$ values for $p p$ at $\sqrt{s}=7 \mathrm{TeV}$ [15] and for $\mathrm{Pb}-\mathrm{Pb}$ at $\sqrt{s_{N N}}=2.76$ and $5.02 \mathrm{TeV}[35,40,58]$ reported by the ALICE Collaboration are represented as a function of $\sqrt{\frac{d N}{d y} / S_{\perp}^{\text {geom }}}$ in Fig. 17. A fourth-degree polynomial function fits rather well the data corresponding to $p p$ at $\sqrt{s}=7 \mathrm{TeV}(\alpha=1)$ and $\mathrm{Pb}-\mathrm{Pb}$ at $\sqrt{s_{N N}}=2.76$ and $5.02 \mathrm{TeV}$. The fit quality is represented 


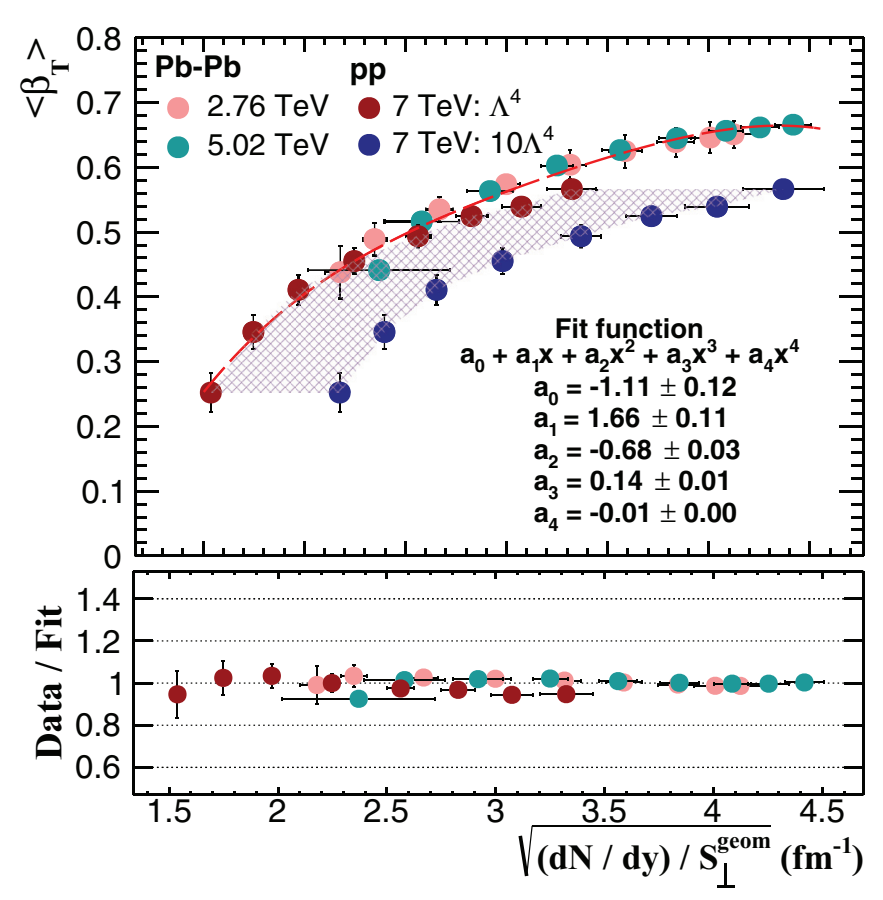

FIG. 17. The BGBW fit parameters $\left\langle\beta_{T}\right\rangle$ as a function of $\sqrt{\frac{d N}{d y} / S_{\perp}^{\text {geom }}}$ for $p p$ at $\sqrt{s}=7 \mathrm{TeV}$ and $\mathrm{Pb}-\mathrm{Pb}$ at $\sqrt{s_{N N}}=2.76$ and 5.02 TeV. The shaded area represents the uncertainty in the overlapping area estimates for $p p$ collisions; see the text.

in the bottom plot of the figure. Qualitatively the trends are similar and there is even a very good quantitative scaling for $\alpha=1$ used in the estimate of $S_{\perp}$ for the $p p$ case. The origin of the remaining differences was discussed above. This similarity shows that the main features of the dynamical evolution of the systems produced in $p p$ or $\mathrm{Pb}-\mathrm{Pb}$ collisions at LHC energies are determined by the density of produced hadrons per unit of rapidity and overlapping area.

\section{CONCLUSIONS}

Based on the data for the highest three energies measured at $\operatorname{RHIC}\left(\sqrt{s_{N N}}=62.4,130,200 \mathrm{GeV}\right)$, the most recent results from BES at RHIC $\left(\sqrt{s_{N N}}=7.7-39 \mathrm{GeV}\right)$, and the highest collision energies at $\mathrm{LHC}\left(\sqrt{s_{N N}}=2.76,5.02 \mathrm{TeV}\right)$, we performed a systematic study of the dependence of different observables on the geometrical variable calculated as the square root of the hadron density per unit of rapidity and unit of overlapping area of two colliding ions. The overlapping area has been estimated in the Glauber MC approach. The experimental $\left\langle p_{T}\right\rangle$ values follow a rather good scaling as a function of this variable for each energy. Linear fits of the experimental data show slopes which increase from pions to protons and decrease from BES to LHC energies. A saturation trend for the most central collisions at LHC is observed. For $\sqrt{s_{N N}}=200 \mathrm{GeV}, 2.76 \mathrm{TeV}$, and $5.02 \mathrm{TeV}$

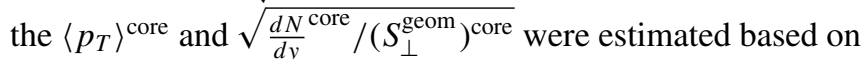
the core-corona approach. The corresponding $\left\langle p_{T}\right\rangle^{\text {core }}$ versus $\sqrt{\frac{d N}{d y}^{\text {core }} /\left(S_{\perp}^{\text {geom }}\right)^{\text {core }}}$ show lower slopes, and their decrease going from $\sqrt{s_{N N}}=200 \mathrm{GeV}$ to $5.02 \mathrm{TeV}$ is less evident for all three species. This shows the importance of discriminating between the corona and core contributions in such a type of analysis, for a quantitative comparison. The decrease in the slopes from RHIC to LHC for all species and for the most central collisions at LHC energies seems to support the approach presented in Ref. [10]. A much better scaling as a function of $\sqrt{\frac{d N}{d y} / S_{\perp}^{\text {geom }}}$ is observed for the slope from the linear fit of the $\left\langle p_{T}\right\rangle$ dependence on the particle mass and the BGBW fit parameter $\left\langle\beta_{T}\right\rangle$. The offset of the $\left\langle p_{T}\right\rangle$ particle mass dependence and the $T_{\text {kin }}^{\text {fo }}$ parameter show a clear jump towards larger values between RHIC and LHC energies. As already mentioned, other phenomena, like suppression and its azimuthal dependence as well as the hydrodynamic expansion in the deconfined and after-hadronization stages, also have to be considered. The very similar dependence of the $\left\langle p_{T}\right\rangle$, $\left\langle p_{T}\right\rangle$ particle mass dependence and the BGBW fit parameter $\left\langle\beta_{T}\right\rangle$ on $\sqrt{\frac{d N}{d y} / S_{\perp}}$ in $p p$ and $\mathrm{Pb}$ - $\mathrm{Pb}$ collisions at LHC energies supports the assumption that the global properties evidenced at LHC energies are determined by the properties of flux tubes of $\sim 1 / \sqrt{\frac{d N}{d y} / S_{\perp}}$ size, the system size playing a minor role.

\section{ACKNOWLEDGMENTS}

This work was carried out under contracts sponsored by the Ministry of Research and Innovation: RONIPALICE04/16.03.2016 (via Institute of Atomic Physics Coordinating Agency) and PN-18 090103.
[1] L. V. Gribov, E. M. Levin, and M. G. Ruskin, Phys. Rep. 100, 1 (1983), and references therein

[2] S. Aid et al. (H1 Collaboration), Nucl. Phys. B 470, 3 (1996).

[3] J. Breitweg et al. (ZEUS Collaboration), Eur. Phys. J. C 7, 609 (1999), and references therein.

[4] F. D. Aaron et al. (H1 Collaboration), Eur. Phys. J. C 64, 561 (2009).

[5] L. McLerran and R. Venugopalan, Phys. Rev. D 49, 2233 (1994); 49, 3352 (1994); 50, 2225 (1994).

[6] E. Iancu, A. Leonidov, and L. McLerran, Nucl. Phys. A 692, 583 (2001); E. Ferreiro, E. Iancu, A. Leonidov, and L. McLerran, ibid. 703, 489 (2002).

[7] D. Kharzeev, E. Levin, and M. Nardi, Nucl. Phys. A 730, 448 (2004); 743, 329 (2004); Phys. Lett. B 561, 93 (2003); Phys. Rev. C 71, 054903 (2005);
D. Kharzeev and M. Nardi, Phys. Lett. B 507, 121 (2001); D. Kharzeev, E. Levin, and L. McLerran, Nucl. Phys. A 748, 627 (2005).

[8] Y. L. Dokshitzer, V. A. Khoze, and S. Troian, J. Phys. G 17, 1585 (1991).

[9] T. Lappi, Eur. Phys. J. C 71, 1699 (2011).

[10] E. Levin and A. H. Rezaeian, Phys. Rev. D 83, 114001 (2011).

[11] V. Khachatryan et al. (CMS Collaboration), J. High Energy Phys. 09 (2010) 091.

[12] S. Chatrchyan et al. (CMS Collaboration), Phys. Lett. B 718, 795 (2013).

[13] B. Abelev et al. (ALICE Collaboration), Phys. Lett. B 719, 29 (2013).

[14] G. Aad et al. (ATLAS Collaboration), Phys. Lett. B 725, 60 (2013). 
[15] C. Andrei (ALICE Collaboration), Nucl. Phys. A 931, c888 (2014).

[16] M. Witek et al. (LHCb Collaboration), EPJ Web Conf. 141, 01007 (2017).

[17] J. Adam et al. (ALICE Collaboration), Nat. Phys. 13, 535 (2017).

[18] E. Shuryak and I. Zahed, Phys. Rev. C 88, 044915 (2013).

[19] H. Petersen et al., Phys. Rev. C 78, 044901 (2008).

[20] W. T. Deng, X. N. Wang, and R. Xu, Phys. Rev. C 83, 014915 (2011).

[21] R. Andrade et al., Eur. Phys. J. A 29, 23 (2006).

[22] J. Xu and C. M. Ko, Phys. Rev. C 84, 014903 (2011).

[23] V. P. Konchakovski et al., Phys. Rev. C 85, 044922 (2012).

[24] T. Pierog et al., Phys. Rev. C 92, 034906 (2015).

[25] T. Sjöstrand, S. Mrenna, and P. Z. Skands, J. High Energy Phys. 05 (2006) 026.

[26] J. Bellm et al., Eur. Phys. J. C 76, 196 (2016).

[27] F. W. Bopp, R. Engel, and J. Ranft, arXiv:hep-ph/9803437.

[28] A. Buckley et al., Phys. Rep. 504, 145 (2011).

[29] C. Bierlich, G. Gustafson, and L. Lönnblad, arXiv:1612.05132 [hep-ph].

[30] R. J. Glauber, Phys. Rev. 100, 242 (1955).

[31] V. Franco and R. J. Glauber, Phys. Rev. 142, 1195 (1966).

[32] M. L. Miller et al., Annu. Rev. Nucl. Part. Sci. 57, 205 (2007).

[33] M. Rybczynski, G. Stefanek, W. Broniowski, and P. Bozek, Comput. Phys. Commun. 185, 1759 (2014).

[34] B. I. Abelev et al. (STAR Collaboration), Phys. Rev. C 79, 034909 (2009).

[35] B. Abelev et al. (ALICE Collaboration), Phys. Rev. C 88, 044910 (2013).

[36] U. Amaldi, 60 Years of CERN Experiments and Discoveries (World Scientific, Singapore, 2015).

[37] J. Adam et al. (ALICE Collaboration), Phys. Rev. Lett. 116, 222302 (2016).

[38] L. Adamczyk et al. (STAR Collaboration), Phys. Rev. C 96, 044904 (2017).

[39] B. Alver et al., Phys. Rev. C 77, 014906 (2008).

[40] N. Jacazio (ALICE Collaboration), Nucl. Phys. A 967, 421 (2017).

[41] X. Zhu (STAR Collaboration), in Critical Point and Onset of Deconfinement, CPOD2011, 7-11.11.2011, Wuhan, China (unpublished).

[42] A. R. Timmins (STAR Collaboration), in Strange Quark Matter Conference, 5-10.10.2008, Beijing, China (unpublished).

[43] M. M. Aggarwal et al. (STAR Collaboration), Phys. Rev. C 83, 024901 (2011).
[44] J. Adams et al. (STAR Collaboration), Phys. Rev. Lett. 98, 062301 (2007).

[45] B. Abelev et al. (ALICE Collaboration), Phys. Rev. Lett. 111, 222301 (2013).

[46] B. Abelev et al., Eur. Phys. J. Web Conf. 171, 13007 (2018).

[47] J. Adam et al. (ALICE Collaboration), Phys. Rev. C 95, 064606 (2017).

[48] F. Becattini et al., Phys. Rev. C 69, 024905 (2004).

[49] P. Bozek, Acta Phys. Polon. B 36, 3071 (2005).

[50] K. Werner, Phys. Rev. Lett. 98, 152301 (2007).

[51] F. Becattini and J. Manninen, J. Phys. G 35, 104013 (2008); Phys. Lett. B 673, 19 (2009).

[52] J. Aichelin and K. Werner, Phys. Rev. C 79, 064907 (2009); J. Phys. G 37, 094006 (2010); Phys. Rev. C 82, 034906 (2010).

[53] P. Bozek, Phys. Rev. C 79, 054901 (2009).

[54] M. Gemard and J. Aichelin, Astron. Nachr. 335, 660 (2014).

[55] M. Petrovici et al., Phys. Rev. C 96, 014908 (2017).

[56] J. Adam et al. (ALICE Collaboration), Eur. Phys. J. C 75, 226 (2015).

[57] A. K. Dash (ALICE Collaboration), in 9th International Workshop on MPI at LHC, December 11-15, 2017, Shimla, India (unpublished).

[58] R. Pregenella (ALICE Collaboration), in International Conference on Strangeness in Quark Matter, SQM, 10-15.10.2017, Utrecht, the Netherlands (unpublished).

[59] E. Schnedermann et al., Phys. Rev. C 48, 2462 (1993).

[60] X. Sun, H. Masui, A. M. Poskanzer, and A. Schman, Phys. Rev. C 91, 024903 (2015).

[61] L. Adamczyk et al. (STAR Collaboration), Phys. Rev. C 93, 014907 (2016).

[62] A. A. Bylinkin and A. A. Rostovtsev, Yad. Fiz. 75, 1060 (2012) [Phys. At. Nuclei 75, 999 (2012)].

[63] A. Bzdak, B. Schenke, P. Tribedy, and R. Venugopalan, Phys. Rev. C 87, 064906 (2013).

[64] B. Schenke, P. Tribedy, and R. Venugopalan, Phys. Rev. C 86, 034908 (2012).

[65] L. McLerran, M. Praszalowicz, and B. Schenke, Nucl. Phys. A 916, 210 (2013).

[66] L. McLerran and M. Praszalowicz, Phys. Lett. B 741, 246 (2015)

[67] T. S. Biro, H. B. Nielsen, and J. Knoll, Nucl. Phys. B 245, 449 (1984).

[68] M. Petrovici et al. (FOPI Collaboration), Phys. Rev. Lett. 74, 5001 (1995), and references therein. 